\title{
A Holistic Approach of Personality Traits in Medical Students: An Integrative Review
}

\author{
Nicoleta Suciu ${ }^{1}$, Lorena Elena Meliț ${ }^{2, *}$ and Cristina Oana Mărginean ${ }^{2}$ D \\ 1 European and Research Projects Department, "George Emil Palade" University of Medicine, Pharmacy, \\ Sciences and Technology, 540136 Târgu Mureș, Romania; nico.suciu03@gmail.com \\ 2 Department of Pediatrics I, "George Emil Palade" University of Medicine, Pharmacy, Sciences and \\ Technology, 540136 Târgu Mureș, Romania; marginean.oana@gmail.com \\ * Correspondence: lory_chimista89@yahoo.com; Tel.: +40-742-984744
}

Citation: Suciu, N.; Meliț, L.E.; Mărginean, C.O. A Holistic Approach of Personality Traits in Medical Students: An Integrative Review. Int. J. Environ. Res. Public Health 2021, 18, 12822. https://doi.org/10.3390/ ijerph182312822

Academic Editor: Florian Fischer

Received: 13 November 2021

Accepted: 2 December 2021

Published: 5 December 2021

Publisher's Note: MDPI stays neutral with regard to jurisdictional claims in published maps and institutional affiliations.

Copyright: (C) 2021 by the authors. Licensee MDPI, Basel, Switzerland. This article is an open access article distributed under the terms and conditions of the Creative Commons Attribution (CC BY) license (https:/ / creativecommons.org/licenses/by/ $4.0 /)$.
Abstract: Personality is one of the most crucial aspects of human life, since it influences all human behaviours in both personal and social life, and might also trigger important conflicts with a person's surroundings in the setting of incompatible traits and characteristics. It is true that 'one must be born' for a certain medical specialty, but several components of personality might be educated with proper training. Increased levels of Conscientiousness, Agreeableness, and Openness associated with lower levels of Neuroticism might represent the key combination for achieving professional satisfaction in the medical profession. Medical students should receive proper interprofessional education, since effective interprofessional relationships among healthcare providers definitely improve patients safety. Empathy contributes to effective patient-physician communication, improving patient trust, compliance, and satisfaction, being positively correlated with Openness, Agreeableness, Conscientiousness and Extraversion. Emotional intelligence-the capacity to respond to one's own and others' emotions-was proven to contribute, in a synergistic way with empathy, to increasing empathic ability. Clinical communication skills represent a key component in medical students in order to achieve the best patient care, and they are certainly related and/or influenced by empathy, interprofessional collaboration skills, emotional intelligence and, especially, personality traits. Taking into account the complex interactions mentioned above, the implementation of effective courses based on these concepts in medical students, intending to promote the development of clinical communication skills, represents a real emergency, since it might result in a reduction in medical errors and subsequent related deaths. A thorough understanding of students' personality is mandatory before designing these courses in order to provide a training tailored to their personality styles.

Keywords: personality; clinical communication; medical students

\section{Introduction}

The ways people think, feel, and behave impact their daily social interactions and should be properly assessed and trained whenever possible, especially in terms of professions that require interhuman interactions. The medical profession is by far the most peculiar of all in terms of communication, since it might act dichotomously resulting in either a strong doctor-patient partnership or, on the contrary, in a poor relationship defined by a lack of trust and respect, which will have a major negative impact on a patient's treatment outcome. However, the importance of medical communication is not always fully acknowledged and valued properly during medical education; once medical students graduate and begin to practice, they become aware of this crucial gap in their education. Moreover, this reflects on their medical actions and decreases their quality considerably. Medical communication should be based on empathy, defined as a complex social emotion consisting of the ability to identify and understand the feelings and thoughts of others and to respond with adequate emotions [1-3]. In terms of the medical profession, the concept of empathy was specified as being defined as a major cognitive attribute that implies the 
quality of understanding a patient's concerns, experiences and perspective, as well as communicating this perception with the aim of helping them [4]. Thus, we emphasise the close interdependence between personality, medical empathy and communication. Medical education should consist of a holistic approach in terms of implementing and monitoring appropriate training strategies for developing proper communication skills, but also for shaping certain personality traits in order to achieve the best level of communication with the future patient. Nevertheless, it is a well-documented fact that personality traits are influenced mostly by genetic background [5], a fact that might represent a real challenge in medical undergraduates, taking into account that genetic inheritance is the most difficult to be modified or trained. Albeit minor, the influence of social skills on the development of personality should not be neglected, since it is the main component that could be modified with proper education.

Personality traits were also proven to be essential for medical students in choosing the most suitable specialty [6] based on a proper assessment and fair acknowledgement of their self-strengths and limitations. This takes into account that professional satisfaction influences not only the doctor's quality of life and performance, but also the patient's outcome. Thus, medical educators should also focus their attention on guiding undergraduates in making the best choice based on their personality and personal interests. Several studies proved that medical students reported personal satisfaction and interest as the most important factors that influenced their decision when choosing their specialty [7]. As additional proof of the complex relationship between personality traits, medical empathy and communication were stated by Hojat et al., who emphasised that a proper understanding of this relationship would not only guide specialty choice, but could also be helpful in selecting students that match the medical profession by predicting possible behaviours [4]. Medical performance represents the top of the pyramid built on personality traits, medical empathy, and communication - the final supreme goal of each physician.

Interprofessional collaboration, teamwork and multidisciplinary approach represent highly desirable skills in the medical profession, taking into account the current medical trends that support and focus on inter- and multidisciplinary medical teams for conveying patient safety, trust, and improving their outcomes [6]. The World Health Organisation emphasised the major importance of interprofessional teamwork in the medical field by implementing medical training focused on developing students' collaboration skills [8]. However, certain personality traits such as neuroticism and introversion might display a negative influence on interprofessional collaboration if not shaped properly during medical school in order to diminish traits that alter this collaboration as much as possible.

Based on these aforementioned facts, we might state that personality traits represent the core of medical performance, irrespective of their chosen specialty, and a thorough understanding and assessment of these traits are the missing puzzle piece for implementing proper medical training and improving patient outcomes.

Thus, the main aim of this narrative overview was to underline the impact of personality traits on a physician's work, academic satisfaction, and their patient's outcome.

\section{Literature Search}

An electronic literature search was performed for the present review on the lines of search for a narrative overview, including three databases: PubMed, Google Scholar and EMBASE. We used the following search terms: 'personality traits', 'medical empathy', 'interprofessional collaboration', 'career choice', 'emotional intelligence', and 'professional satisfaction' (Figure 1). We should mention that Google Scholar was an auxiliary database used if the full text of a particular article was not found in PubMed or Embase. The inclusion criteria consisted of all types of articles that involved medical students, young residents or physicians, irrespective of the year of publication, in order to also highlight the progress of medical education in terms of acknowledging the importance of personality traits in the professional development of medical students. Each of the included articles was critically assessed according to the following: key results, quality of the results, interpretation of the 
results, suitability of the methods used to verify the hypothesis, limitations, and impact of conclusions to the field [9]. Moreover, we applied critical reading tools for scientific articles according to Jean-Baptist du Prel et al. [10], by analysing the study design of each article, the structure, the role of each section, as well as the potential sources of bias and limitations. Thus, analysis and inference were the most reliable critical reading tools used for the assessment of articles included in this review. We excluded articles that did not fit to the aforementioned criteria, as well as articles that were not written in English and those for which the full text was not available. We also identified additional references that fulfilled our criteria by a manual search of the reference list from the articles retrieved in the first round of search, for which we followed the same steps before including them in the review. We synthetized the findings from the remaining articles, highlighting both the similarities and inconsistencies between them in order to provide an objective narrative overview related to the impact of personality traits on a physician's work, academic satisfaction, and their patient's outcome. The methodological characteristics of the research-type studies included in this review are described in Table 1.

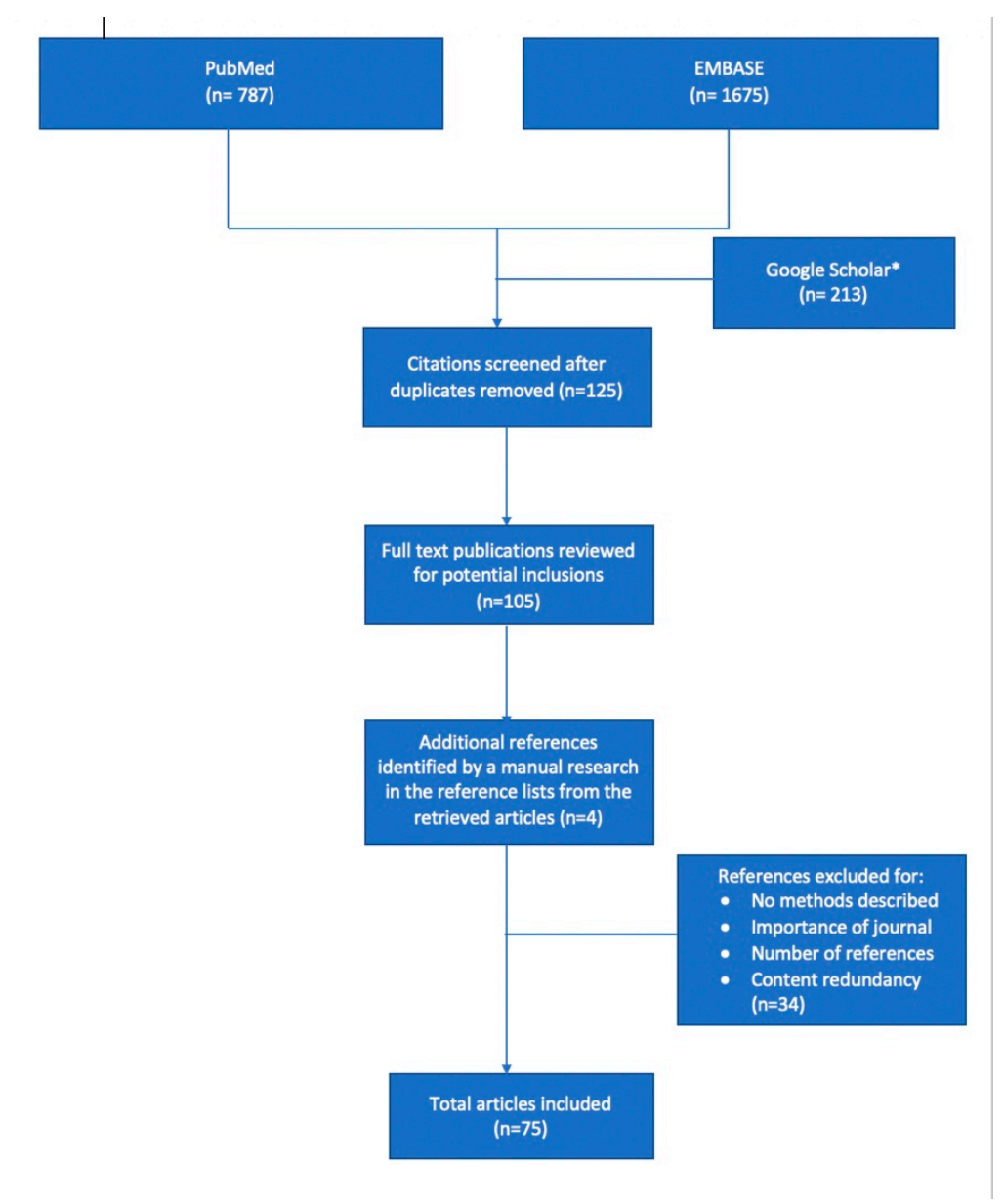

Figure 1. Flowchart of the literature selection. * Google Scholar was an auxiliary database used if the full text of a particular article was not found in PubMed or Embase. 
Table 1. The methodological characteristics of the research-type studies included in this review.

\begin{tabular}{|c|c|c|c|c|c|c|c|}
\hline $\begin{array}{c}\text { Author, Year, and } \\
\text { Country }\end{array}$ & Title of the Study & Aim & Study Type & Sample & $\begin{array}{c}\text { Median Age or Mean } \\
\text { (Years)/Gender (\%) }\end{array}$ & Methods/Scales & Observations \\
\hline $\begin{array}{l}\text { Guilera et al., 2019, } \\
\text { Spain [1] }\end{array}$ & $\begin{array}{l}\text { Empathy and big five } \\
\text { personality model in medical } \\
\text { students and its relationship } \\
\text { to gender and specialty } \\
\text { preference: a cross-sectional } \\
\text { study. }\end{array}$ & $\begin{array}{l}\text { To explore the relation-ship } \\
\text { between empathy and } \\
\text { personality }\end{array}$ & $\begin{array}{c}\text { Prospective } \\
\text { cross-sectional study) }\end{array}$ & $\begin{array}{l}110 \text { medical } \\
\text { students }\end{array}$ & $22 / 76.4 \% \mathrm{~F}$ & $\begin{array}{ll}\bullet & \text { JSPE } \\
\bullet & \text { IRI } \\
\bullet & \text { NEO-FFI }\end{array}$ & $\begin{array}{l}\text { Empathy is related to } \\
\text { personality }\end{array}$ \\
\hline $\begin{array}{l}\text { Baron-Cohen et al., } \\
\text { 2004, United } \\
\text { Kingdom [2] }\end{array}$ & $\begin{array}{l}\text { The empathy quotient: an } \\
\text { investigation of adults with } \\
\text { Asperger syndrome or high } \\
\text { functioning autism, and } \\
\text { normal sex differences }\end{array}$ & $\begin{array}{l}\text { To test EQ score in } \\
\text { high-functioning autism } \\
\text { (HFA) and Asperger } \\
\text { Syndrome (AS) }\end{array}$ & $\begin{array}{c}\text { Prospective } \\
\text { case-control study }\end{array}$ & $\begin{array}{c}180 \\
\text { high-functioning } \\
\text { autism (HFA) and } \\
\text { Asperger } \\
\text { Syndrome (AS) } \\
\text { subjects }\end{array}$ & $34.2 / 72.22 \% \mathrm{M}$ & $\begin{array}{ll}\bullet & \text { EQ } \\
\bullet & \text { WAIS-R }\end{array}$ & $\begin{array}{c}\text { Empathy deficit in } \\
\text { AS/HFA }\end{array}$ \\
\hline $\begin{array}{l}\text { Hojat et al., 2015, } \\
\text { USA [4] }\end{array}$ & $\begin{array}{l}\text { Eleven years of data on the } \\
\text { Jefferson scale of } \\
\text { empathy-medical student } \\
\text { version (JSE-S): proxy norm } \\
\text { data and tentative cutoff } \\
\text { scores }\end{array}$ & $\begin{array}{l}\text { To obtain cutoff scores for } \\
\text { the JSE S-version }\end{array}$ & $\begin{array}{c}\text { Prospective } \\
\text { observational study }\end{array}$ & $\begin{array}{l}2637 \text { medical } \\
\text { students }\end{array}$ & $23.4 \pm 2.4 \mathrm{SD} / 50.66 \% \mathrm{~F}$ & JSE-S & $\begin{array}{l}\text { Usefulness of empathy } \\
\text { in decision making }\end{array}$ \\
\hline $\begin{array}{l}\text { Yusoff et al., 2018, } \\
\text { Malaysia [5] }\end{array}$ & $\begin{array}{l}\text { Which personality traits have } \\
\text { favourable impact on } \\
\text { psychological health during } \\
\text { stressful condition? }\end{array}$ & $\begin{array}{l}\text { To investigate which } \\
\text { personality traits have a } \\
\text { favourable impact on the } \\
\text { psychological health of } \\
\text { medical students during the } \\
\text { most stressful period }\end{array}$ & $\begin{array}{c}\text { Prospective } \\
\text { cross-sectional study }\end{array}$ & $\begin{array}{l}174 \text { medical } \\
\text { students }\end{array}$ & $19.27 / 67.2 \% \mathrm{~F}$ & $\begin{array}{l}\text { - } \quad \text { Personality type } \\
\text { DASS-21 } \\
\text { USMaP-i }\end{array}$ & $\begin{array}{l}\text { - NEO-FFI } \\
\text { Favourable impact on } \\
\text { neuroticism, } \\
\text { unfavourable impact on } \\
\text { stress }\end{array}$ \\
\hline $\begin{array}{l}\text { Kwon et al., 2016, } \\
\text { Korea [6] }\end{array}$ & $\begin{array}{l}\text { Specialty Choice Preference of } \\
\text { Medical Students According } \\
\text { to Personality Traits by } \\
\text { Five-Factor Model. }\end{array}$ & $\begin{array}{c}\text { To evaluate personality by } \\
\text { NEO-FFI }\end{array}$ & $\begin{array}{c}\text { Prospective } \\
\text { cross-sectional study }\end{array}$ & $\begin{array}{l}110 \text { medical } \\
\text { students }\end{array}$ & $\begin{array}{c}28.9 \pm 2.1 \mathrm{SD} / \\
39 \% \mathrm{~F}\end{array}$ & $\begin{array}{l}\text { - } \quad \text { Personality traits } \\
\text { by NEO-FFI }\end{array}$ & $\begin{array}{l}\text { Personality important } \\
\text { in choosing medical } \\
\text { specialty }\end{array}$ \\
\hline $\begin{array}{l}\text { McCrae et al., 1989, } \\
\text { USA [7] }\end{array}$ & $\begin{array}{l}\text { Reinterpreting the } \\
\text { Myers-Briggs Type Indicator } \\
\text { from the Perspective of the } \\
\text { Five-Factor Model of } \\
\text { Personality. }\end{array}$ & $\begin{array}{l}\text { The MBTI indicator was } \\
\text { evaluated from the } \\
\text { perspectives of Jung's } \\
\text { theory of psychological } \\
\text { types and the five-factor } \\
\text { model of personality on the } \\
\text { NEO-PI }\end{array}$ & $\begin{array}{c}\text { Prospective } \\
\text { observational } \\
\text { longitudinal study }\end{array}$ & 468 adults & $\begin{array}{c}62.7 \mathrm{M} \text { and } 58.9 \mathrm{~F} / 57.05 \% \\
\mathrm{M}\end{array}$ & $\begin{array}{ll}\bullet & \text { MBTI indicator } \\
- & \text { NEO-PI } \\
& \text { Psychological } \\
& \text { types }\end{array}$ & $\begin{array}{l}\text { To reinterpret the MBTI } \\
\text { in terms of the } \\
\text { five-factor model }\end{array}$ \\
\hline
\end{tabular}


Table 1. Cont.

\begin{tabular}{|c|c|c|c|c|c|c|c|}
\hline $\begin{array}{l}\text { Author, Year, and } \\
\text { Country }\end{array}$ & Title of the Study & Aim & Study Type & Sample & $\begin{array}{c}\text { Median Age or Mean } \\
\text { (Years)/Gender (\%) }\end{array}$ & Methods/Scales & Observations \\
\hline $\begin{array}{l}\text { Olsson et al., 2020, } \\
\text { Sweden [8] }\end{array}$ & $\begin{array}{l}\text { Personality and Learning } \\
\text { Styles in Relation to Attitudes } \\
\text { towards Interprofessional } \\
\text { Education: A Cross-Sectional } \\
\text { Study on Undergraduate } \\
\text { Medical Students during Their } \\
\text { Clinical Courses }\end{array}$ & $\begin{array}{l}\text { To investigate the effect of } \\
\text { personality traits and } \\
\text { learning styles on medical } \\
\text { students' attitudes towards } \\
\text { IPE }\end{array}$ & $\begin{array}{c}\text { Prospective } \\
\text { cross-sectional study }\end{array}$ & $\begin{array}{l}79 \text { medical } \\
\text { students }\end{array}$ & $29 / 63 \% \mathrm{~F}$ & $\begin{array}{r}\text { NEO-FFI } \\
\text { IPE }\end{array}$ & $\begin{array}{l}\text { No correlation between } \\
\text { personality, learning } \\
\text { style and attitude } \\
\text { towards IPE }\end{array}$ \\
\hline $\begin{array}{l}\text { Tamannaeifar et al., } \\
\text { 2014, Iran [11] }\end{array}$ & $\begin{array}{l}\text { The relationship between } \\
\text { personality characteristics, } \\
\text { social support and life } \\
\text { satisfaction with university } \\
\text { students' academic } \\
\text { performance }\end{array}$ & $\begin{array}{c}\text { To investigate the } \\
\text { relationship among } \\
\text { personality characteristics, } \\
\text { social support and life } \\
\text { satisfaction with academic } \\
\text { performance } \\
\end{array}$ & $\begin{array}{c}\text { Prospective } \\
\text { descriptive, } \\
\text { correlative survey }\end{array}$ & $\begin{array}{l}250 \text { humanities' } \\
\text { students }\end{array}$ & $-/-$ & $\begin{array}{r}-\quad \text { NEO-FFI } \\
\text { SWLSSS }\end{array}$ & $\begin{array}{l}\text { Relationship between } \\
\text { neuroticism and } \\
\text { conscientiousness with } \\
\text { academic performance }\end{array}$ \\
\hline $\begin{array}{l}\text { Nasri et al., 2017, } \\
\text { Iran [12] }\end{array}$ & $\begin{array}{l}\text { Personality characteristics, } \\
\text { irrational beliefs, and } \\
\text { communication skills as } \\
\text { predictors school counselors' } \\
\text { job performance. }\end{array}$ & $\begin{array}{l}\text { To predict counsellors' job } \\
\text { performance based on their } \\
\text { personality }\end{array}$ & $\begin{array}{l}\text { Prospective clustering } \\
\text { random sampling } \\
\text { method }\end{array}$ & $\begin{array}{l}283 \text { counsellors } \\
\text { working }\end{array}$ & $-/-$ & - $\quad$ NEO-FFI & $\begin{array}{c}\text { Personality } \\
\text { characteristics are } \\
\text { predictors of } \\
\text { counsellors' job } \\
\text { performance }\end{array}$ \\
\hline $\begin{array}{l}\text { Vassend et al., 2011, } \\
\text { USA [13] }\end{array}$ & $\begin{array}{l}\text { The NEO personality } \\
\text { inventory revised (NEO-PI-R): } \\
\text { Exploring the measurement } \\
\text { structure and variants of the } \\
\text { five-factor model }\end{array}$ & $\begin{array}{l}\text { To evaluate NEO-PI-R } \\
\text { personality }\end{array}$ & Prospective study & 856 adults & $50.8 / 55.84 \% \mathrm{~F}$ & NEO-PI-R & $\begin{array}{l}\text { NEO-PI-R reflects } \\
\text { personality }\end{array}$ \\
\hline $\begin{array}{l}\text { Lievens et al., 2002, } \\
\text { Belgium [14] }\end{array}$ & $\begin{array}{l}\text { Medical students' personality } \\
\text { characteristics and academic } \\
\text { performance: a five-factor } \\
\text { model perspective }\end{array}$ & $\begin{array}{l}\text { To evaluate NEO-PI-R } \\
\text { personality }\end{array}$ & $\begin{array}{c}\text { Prospective } \\
\text { cross-sectional } \\
\text { inventory study and } \\
\text { prospective } \\
\text { longitudinal study of } \\
\text { one cohort of medical } \\
\text { students }\end{array}$ & $\begin{array}{l}785 \text { Flemish } \\
\text { medical students } \\
\text { and } 1361 \text { students } \\
\text { from Ghent } \\
\text { University }\end{array}$ & $18.2 / 60 \% \mathrm{~F}$ & NEO-PI-R & $\begin{array}{l}\text { Personality } \\
\text { assessment-a useful } \\
\text { tool in student } \\
\text { counselling and } \\
\text { guidance }\end{array}$ \\
\hline $\begin{array}{l}\text { Han et a., 2017, } \\
\text { USA [15] }\end{array}$ & $\begin{array}{l}\text { Big five personality factors } \\
\text { and facets as predictors of } \\
\text { openness to diversity }\end{array}$ & $\begin{array}{l}\text { To examine the associations } \\
\text { between NEO-FFI higher } \\
\text { order factors and lower } \\
\text { order factors and } \\
\text { universal-diverse } \\
\text { orientation }\end{array}$ & $\begin{array}{c}\text { Prospective } \\
\text { cross-sectional study }\end{array}$ & $\begin{array}{l}\text { Study } 1-388 \\
\text { medical students } \\
\text { and Study } 2-176 \\
\text { undergraduates }\end{array}$ & $\begin{array}{l}21.18 / 58.6 \% \text { F-study } 1 \\
21.01 / 72.2 \% \text { F-study } 2\end{array}$ & $\begin{array}{r}\text { NEO-FFI } \\
\text { UDO }\end{array}$ & $\begin{array}{l}\text { Practical implications } \\
\text { on how personality } \\
\text { factors are incorporated } \\
\text { into current diversity } \\
\text { interventions }\end{array}$ \\
\hline $\begin{array}{l}\text { Borges et al., 2008, } \\
\text { USA [16] }\end{array}$ & $\begin{array}{l}\text { Emotional intelligence and } \\
\text { medical specialty choice: } \\
\text { findings from three empirical } \\
\text { studies }\end{array}$ & $\begin{array}{l}\text { To examine emotional } \\
\text { intelligence (EI) and } \\
\text { specialty choice among } \\
\text { students at three US medical } \\
\text { schools }\end{array}$ & $\begin{array}{c}\text { Prospective } \\
\text { observational study }\end{array}$ & $\begin{array}{c}84 \text { medical } \\
\text { students—study } 1 \\
250 \text { medical } \\
\text { students—study } 2 \\
292 \text { medical } \\
\text { students—study } 3\end{array}$ & $\begin{array}{c}24.3 / 50 \% \text { F-study } 1 \\
22.7 / 44.8 \% \text { F-study } 2 \\
23.6 / 38 \% \text { F-study } 2\end{array}$ & $\begin{array}{l}\text { - } \quad \text { MSCEIT }{ }^{\mathrm{TM}} \text { study } \\
1 \\
\text { - } \\
\text { TMMS and IRI, } \\
\text { study } 2 \\
\text { EQ-I }{ }^{\circledR} \text {, study } 3\end{array}$ & $\begin{array}{c}\text { No significant } \\
\text { differences in EI } \\
\text { between students } \\
\text { entering primary care } \\
\text { and non-primary care } \\
\text { specialties }\end{array}$ \\
\hline
\end{tabular}


Table 1. Cont.

\begin{tabular}{|c|c|c|c|c|c|c|c|}
\hline $\begin{array}{c}\text { Author, Year, and } \\
\text { Country }\end{array}$ & Title of the Study & Aim & Study Type & Sample & $\begin{array}{l}\text { Median Age or Mean } \\
\text { (Years)/Gender (\%) }\end{array}$ & Methods/Scales & Observations \\
\hline $\begin{array}{l}\text { Hojat et al., 2008, } \\
\text { USA [17] }\end{array}$ & $\begin{array}{l}\text { Personality and specialty } \\
\text { interest in medical students }\end{array}$ & $\begin{array}{l}\text { To evaluate the impact of } \\
\text { personality score on career } \\
\text { interests }\end{array}$ & $\begin{array}{c}\text { Prospective } \\
\text { observational study }\end{array}$ & $\begin{array}{l}1076 \text { medical } \\
\text { students }\end{array}$ & $-/ 64 \% \mathrm{M}$ & ZKPQ-S & $\begin{array}{l}\text { Personalities of medical } \\
\text { students predict their } \\
\text { career interests }\end{array}$ \\
\hline $\begin{array}{l}\text { McGreevy et al., } \\
\text { 2002, USA [19] }\end{array}$ & $\begin{array}{l}\text { Preliminary measurement of } \\
\text { the surgical personality. }\end{array}$ & $\begin{array}{l}\text { To test for a distinct surgical } \\
\text { personality }\end{array}$ & $\begin{array}{c}\text { Prospective } \\
\text { observational study }\end{array}$ & $\begin{array}{l}39 \text { surgical } \\
\text { residents }\end{array}$ & $/ 61.53 \% \mathrm{M}$ & NEO-PI-R & $\begin{array}{l}\text { Similarity of trait } \\
\text { variance from the } \\
\text { general population in } \\
\text { both male and female } \\
\text { surgical residents }\end{array}$ \\
\hline $\begin{array}{c}\text { Hoffman et al., 2010, } \\
\text { United Kingdom } \\
\text { [20] }\end{array}$ & $\begin{array}{l}\text { Personality differences } \\
\text { between surgery residents, } \\
\text { nonsurgery residents, and } \\
\text { medical students }\end{array}$ & $\begin{array}{l}\text { To examine specialty group } \\
\text { differences in personality } \\
\text { traits }\end{array}$ & $\begin{array}{c}\text { Prospective } \\
\text { observational study }\end{array}$ & $\begin{array}{l}274 \text { surgical } \\
\text { residents and } 207 \\
\text { medical students }\end{array}$ & $/ 53 \% \mathrm{M}$ & OCEAN personality & $\begin{array}{c}\text { Greater levels of } \\
\text { conscientiousness were } \\
\text { observed in surgery } \\
\text { residents }\end{array}$ \\
\hline $\begin{array}{l}\text { Preece et al., 2016, } \\
\text { United Kingdom } \\
\text { [21] }\end{array}$ & $\begin{array}{c}\text { Are surgeons born or made? } \\
\text { A comparison of personality } \\
\text { traits and learning styles } \\
\text { between surgical trainees and } \\
\text { medical students }\end{array}$ & $\begin{array}{l}\text { To score } 5 \text { personality } \\
\text { domains (extraversion, } \\
\text { conscientiousness, } \\
\text { agreeableness, openness to } \\
\text { experience, and } \\
\text { neuroticism) }\end{array}$ & $\begin{array}{c}\text { Prospective } \\
\text { cross-sectional study }\end{array}$ & $\begin{array}{l}53 \text { medical } \\
\text { students and } 37 \\
\text { surgical trainees }\end{array}$ & $-/-$ & FFM & $\begin{array}{l}\text { Similarities in the } \\
\text { personality traits and } \\
\text { learning styles of } \\
\text { surgical trainees and } \\
\text { students interested in } \\
\text { surgical career }\end{array}$ \\
\hline $\begin{array}{l}\text { Foster et al., 2010, } \\
\text { USA [22] }\end{array}$ & $\begin{array}{l}\text { A psychological profile of } \\
\text { surgeons and surgical } \\
\text { residents }\end{array}$ & $\begin{array}{l}\text { To determine work-related } \\
\text { personality and interest } \\
\text { variables }\end{array}$ & $\begin{array}{c}\text { Prospective } \\
\text { observational study }\end{array}$ & $\begin{array}{l}63 \text { surgical } \\
\text { residents and } 27 \\
\text { attend-- } \\
\text { ing/teaching } \\
\text { surgeons }\end{array}$ & $\begin{array}{l}\text { - } \quad 31.2 / 74.60 \% \mathrm{M} \text { sur- } \\
\text { gical residents } \\
\text { 49.3/ 92.59\% M teaching } \\
\text { surgeons }\end{array}$ & $\begin{array}{l}-\quad \text { CIA } \\
\text { JSI }\end{array}$ & $\begin{array}{l}\text { WOWI Online } \\
\text { assessment tool } \\
\text { provides a stable profile } \\
\text { of successful surgeons }\end{array}$ \\
\hline $\begin{array}{l}\text { Hayashi et al., 2012, } \\
\text { Japan [23] }\end{array}$ & $\begin{array}{l}\text { Changes in attitudes toward } \\
\text { interprofessional health care } \\
\text { teams and education in the } \\
\text { first- and third-year } \\
\text { undergraduate students }\end{array}$ & $\begin{array}{c}\text { To assess the } \\
\text { implementation of a lecture } \\
\text { style for 1st year students } \\
\text { and a training style for 3rd } \\
\text { year students }\end{array}$ & $\begin{array}{c}\text { Prospective } \\
\text { observational study }\end{array}$ & $\begin{array}{l}285 \text { medical } \\
\text { students }\end{array}$ & $-/-$ & $\begin{array}{r}\text { - } \quad \text { ATHCTS } \\
\text { RIPLS }\end{array}$ & $\begin{array}{l}\text { Scores improved after } \\
\text { the training-style } \\
\text { learning approach was } \\
\text { implemented in the } \\
\text { third-year students }\end{array}$ \\
\hline $\begin{array}{l}\text { Wilhelmsson et al., } \\
\text { 2011, Sweden [24] }\end{array}$ & $\begin{array}{l}\text { Are female students in general } \\
\text { and nursing students more } \\
\text { ready for teamwork and } \\
\text { interprofessional collaboration } \\
\text { in healthcare? }\end{array}$ & $\begin{array}{l}\text { To investigate if student } \\
\text { characteristics have an } \\
\text { impact on their } \\
\text { open-mindedness about } \\
\text { cooperation with other } \\
\text { professionals }\end{array}$ & $\begin{array}{l}\text { Prospective } \\
\text { cross-sectional study }\end{array}$ & $\begin{array}{l}670 \text { medical } \\
\text { students }\end{array}$ & $-/ 73.1 \% \mathrm{~F}$ & RIPLS & $\begin{array}{l}\text { Indicates some } \\
\text { directions for more } \\
\text { successful } \\
\text { interprofessional } \\
\text { education }\end{array}$ \\
\hline $\begin{array}{c}\text { Alghasham et al., } \\
\text { 2021, Saudi Arabia } \\
\text { [25] }\end{array}$ & $\begin{array}{l}\text { Effect of students' learning } \\
\text { styles on classroom } \\
\text { performance in } \\
\text { problem-based learning }\end{array}$ & To identify learning styles & $\begin{array}{c}\text { Prospective } \\
\text { observational study }\end{array}$ & $\begin{array}{l}65 \text { medical } \\
\text { students }\end{array}$ & $-/ 73.84 \% \mathrm{M}$ & $\begin{array}{l}\text { Learning Style } \\
\text { Inventory } \\
\text { Questionnaire }\end{array}$ & $\begin{array}{l}\text { Students should be } \\
\text { informed about their } \\
\text { preferred learning style }\end{array}$ \\
\hline
\end{tabular}


Table 1. Cont.

\begin{tabular}{|c|c|c|c|c|c|c|c|}
\hline $\begin{array}{l}\text { Author, Year, and } \\
\text { Country }\end{array}$ & Title of the Study & Aim & Study Type & Sample & $\begin{array}{l}\text { Median Age or Mean } \\
\text { (Years)/Gender (\%) }\end{array}$ & Methods/Scales & Observations \\
\hline $\begin{array}{l}\text { Tariq et al., 2016, } \\
\text { Pakistan [26] }\end{array}$ & $\begin{array}{l}\text { Association between academic } \\
\text { learning strategies and annual } \\
\text { examination results among } \\
\text { medical students of King } \\
\text { Edward Medical University }\end{array}$ & $\begin{array}{l}\text { To find an association } \\
\text { between academic learning } \\
\text { strategies and annual } \\
\text { examination }\end{array}$ & $\begin{array}{l}\text { Prospective simple } \\
\text { random sampling }\end{array}$ & $\begin{array}{l}300 \text { medical } \\
\text { students }\end{array}$ & $-/ 56 \% \mathrm{~F}$ & $\begin{array}{l}\text { Biodata pro forma } \\
\text { ILS questionnaire }\end{array}$ & $\begin{array}{l}\text { Females outperform } \\
\text { their male counterparts } \\
\text { in academic } \\
\text { performance }\end{array}$ \\
\hline $\begin{array}{l}\text { Avrech Bar et al., } \\
\text { 2018, Israel [27] }\end{array}$ & $\begin{array}{l}\text { The role of personal resilience } \\
\text { and personality traits of } \\
\text { healthcare students on their } \\
\text { attitudes towards } \\
\text { interprofessional } \\
\text { collaboration. }\end{array}$ & $\begin{array}{l}\text { To examine the attitudes of } \\
\text { nursing, occupational } \\
\text { therapy and physical } \\
\text { therapy students towards } \\
\text { interprofessional } \\
\text { collaboration }\end{array}$ & $\begin{array}{c}\text { Prospective } \\
\text { descriptive } \\
\text { cross-sectional study }\end{array}$ & $\begin{array}{l}184 \text { healthcare } \\
\text { students }\end{array}$ & $25.4 \pm 2.8 / 16.8 \% \mathrm{M}$ & $\begin{array}{l}\bullet \quad \text { IEPS } \\
\text { CD-RISC } \\
\text { BFI }\end{array}$ & $\begin{array}{l}\text { IPE, including PBL, } \\
\text { should be integrated in } \\
\text { health profession } \\
\text { students' training }\end{array}$ \\
\hline $\begin{array}{l}\text { Hojat et al., 2011, } \\
\text { USA [28] }\end{array}$ & $\begin{array}{l}\text { Empathic and sympathetic } \\
\text { orientations toward patient } \\
\text { care: conceptualization, } \\
\text { measurement, and } \\
\text { psychometrics. }\end{array}$ & $\begin{array}{l}\text { To develop instruments for } \\
\text { measuring empathic and } \\
\text { sympathetic orientations in } \\
\text { patient care and to provide } \\
\text { evidence in support of their } \\
\text { psychometrics }\end{array}$ & $\begin{array}{c}\text { Prospective } \\
\text { observational study }\end{array}$ & $\begin{array}{l}201 \text { medical } \\
\text { students }\end{array}$ & $-/-$ & $\begin{array}{l}\text { - } \quad \text { JSE } \\
\text { IRI } \\
\text { Empathic and } \\
\text { Sympathetic Care }\end{array}$ & $\begin{array}{l}\text { The validated measures } \\
\text { of empathic and } \\
\text { sympathetic orientation } \\
\text { provide research } \\
\text { opportunities }\end{array}$ \\
\hline $\begin{array}{l}\text { Abe et al., 2018, } \\
\text { Japan [29] }\end{array}$ & $\begin{array}{l}\text { Associations between } \\
\text { emotional intelligence, } \\
\text { empathy and personality in } \\
\text { Japanese Medical Students. }\end{array}$ & $\begin{array}{l}\text { To investigate: } \\
\text { (1) The association between } \\
\text { empathy, EI, and personality } \\
\text { (2) Gender differences in the } \\
\text { association between } \\
\text { empathy, EI, and } \\
\text { personality }\end{array}$ & $\begin{array}{c}\text { Prospective } \\
\text { observational study }\end{array}$ & $\begin{array}{l}351 \text { medical } \\
\text { students }\end{array}$ & $20.42 / 70 \% \mathrm{M}$ & $\begin{array}{l}\text { - } \quad \text { TEIQue-SF } \\
\text { JSPE } \\
\text { NEO-FFI }\end{array}$ & $\begin{array}{l}\text { Medical students' EI } \\
\text { may be enhanced with } \\
\text { thoughtful training }\end{array}$ \\
\hline $\begin{array}{l}\text { Costa et al., 2014, } \\
\text { Portugal [30] }\end{array}$ & $\begin{array}{l}\text { Associations between medical } \\
\text { student empathy and } \\
\text { personality: a } \\
\text { multi-institutional study. }\end{array}$ & $\begin{array}{l}\text { To assess associations across } \\
\text { institutions, looking for } \\
\text { personality differences } \\
\text { between students with high } \\
\text { empathy and low empathy } \\
\text { levels }\end{array}$ & $\begin{array}{c}\text { Prospective } \\
\text { observational study }\end{array}$ & $\begin{array}{l}472 \text { medical } \\
\text { students }\end{array}$ & $21 / 66.10 \% \mathrm{~F}$ & $\begin{array}{c}\text { NEO-FFI } \\
\text { JSPE }\end{array}$ & $\begin{array}{l}\text { Medical schools may } \\
\text { need to pay attention to } \\
\text { the personality of } \\
\text { medical students }\end{array}$ \\
\hline $\begin{array}{l}\text { Chew et al., 2013, } \\
\text { Malaysia [31] }\end{array}$ & $\begin{array}{l}\text { Emotional intelligence and } \\
\text { academic performance in first } \\
\text { and final year medical } \\
\text { students: a cross-sectional } \\
\text { study }\end{array}$ & $\begin{array}{l}\text { To examine the effect of EI } \\
\text { on academic performance in } \\
\text { first- and final-year medical } \\
\text { students }\end{array}$ & $\begin{array}{c}\text { Prospective } \\
\text { cross-sectional study }\end{array}$ & $\begin{array}{l}163 \text { medical } \\
\text { students }\end{array}$ & $21.8 \pm 1.98 / 68.7 \% \mathrm{~F}$ & MSCEIT & $\begin{array}{l}\text { Emotional skill } \\
\text { development may } \\
\text { enhance medical } \\
\text { students' academic } \\
\text { performance }\end{array}$ \\
\hline $\begin{array}{l}\text { Aithal et al., 2016, } \\
\text { India [32] }\end{array}$ & $\begin{array}{l}\text { A survey-based study of } \\
\text { emotional intelligence as it } \\
\text { relates to gender and } \\
\text { academic performance of } \\
\text { medical students }\end{array}$ & $\begin{array}{l}\text { To assess trait EI, to examine } \\
\text { possible differences in EI } \\
\text { level, and to establish a } \\
\text { correlation between the EI } \\
\text { of medical students and } \\
\text { their academic performance }\end{array}$ & $\begin{array}{c}\text { Prospective } \\
\text { cross-sectional survey }\end{array}$ & $\begin{array}{c}200 \\
\text { undergraduate } \\
\text { medical students }\end{array}$ & -/107 F/95 M & TEIQue-SF & $\begin{array}{l}\text { Positive correlation } \\
\text { between EI and } \\
\text { academic performance }\end{array}$ \\
\hline
\end{tabular}


Table 1. Cont.

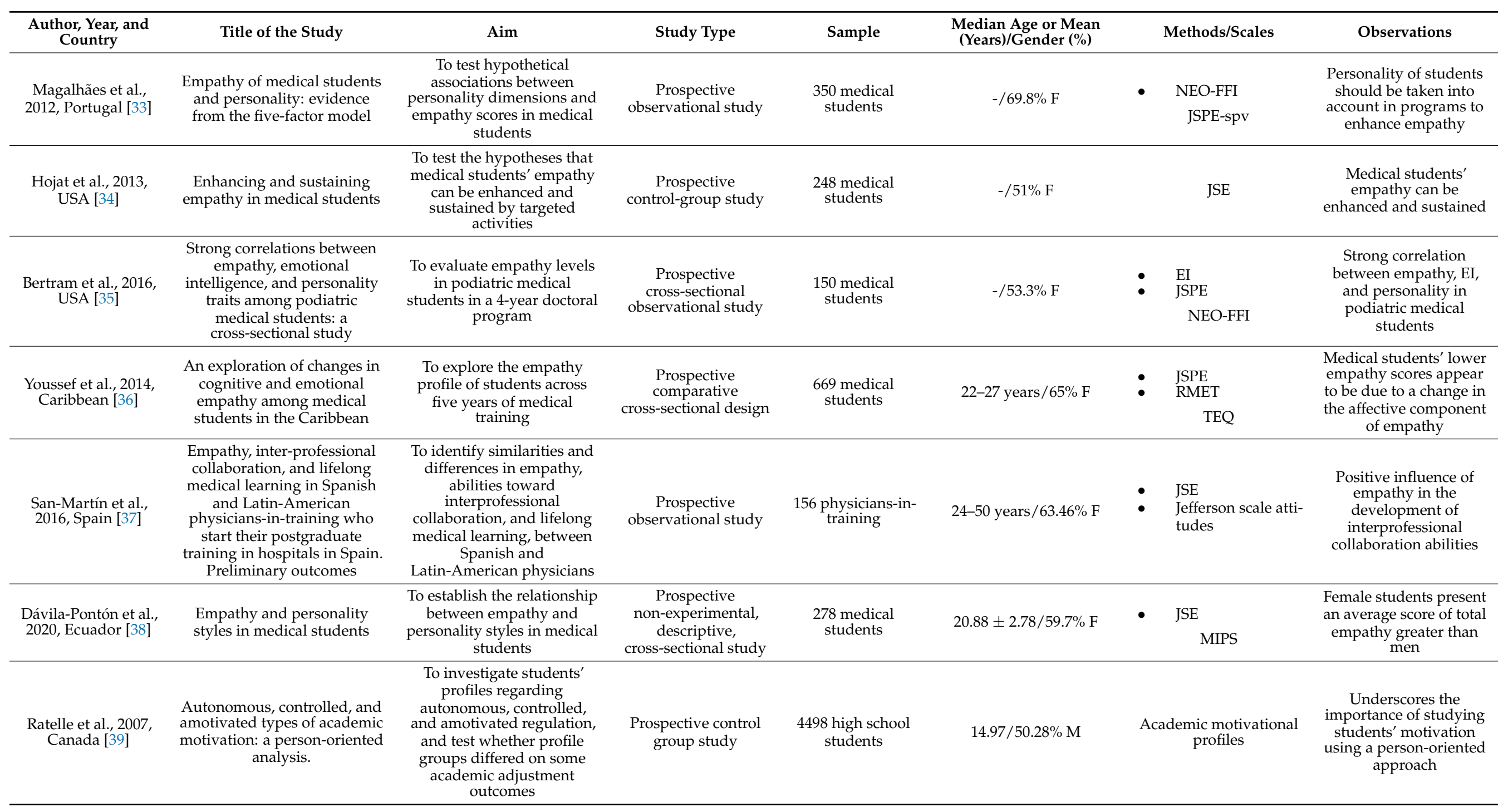


Table 1. Cont.

\begin{tabular}{|c|c|c|c|c|c|c|c|}
\hline $\begin{array}{l}\text { Author, Year, and } \\
\text { Country }\end{array}$ & Title of the Study & Aim & Study Type & Sample & $\begin{array}{l}\text { Median Age or Mean } \\
\text { (Years)/Gender (\%) }\end{array}$ & Methods/Scales & Observations \\
\hline $\begin{array}{c}\text { Doménech-Betoret } \\
\text { et al., 2017, Spain } \\
\text { [40] }\end{array}$ & $\begin{array}{l}\text { Self-Efficacy, satisfaction, and } \\
\text { academic achievement: the } \\
\text { mediator role of students' } \\
\text { expectancy-value beliefs. }\end{array}$ & $\begin{array}{l}\text { To examine and identify } \\
\text { underlying motivational } \\
\text { processes through which } \\
\text { students' academic } \\
\text { self-efficacy affects student } \\
\text { achievement and } \\
\text { satisfaction }\end{array}$ & $\begin{array}{c}\text { Prospective } \\
\text { observational study }\end{array}$ & $\begin{array}{l}797 \text { secondary } \\
\text { education } \\
\text { students }\end{array}$ & $12-17$ years/ $50.7 \% \mathrm{M}$ & $\begin{array}{l}\text { - } \quad \text { Self-efficacy } \\
\text { The achieve- } \\
\text { ment/satisfaction } \\
\text { relationship }\end{array}$ & $\begin{array}{c}\text { Students' expectancy } \\
\text { value beliefs played a } \\
\text { mediator role between } \\
\text { academic self-efficacy } \\
\text { and the achieve- } \\
\text { ment/satisfaction } \\
\text { relationship }\end{array}$ \\
\hline $\begin{array}{l}\text { Webb et al., 2010, } \\
\text { USA [41] }\end{array}$ & $\begin{array}{l}\text { Emotional Intelligence and the } \\
\text { ACGME Competencies. }\end{array}$ & $\begin{array}{l}\text { To evaluate the use of EI } \\
\text { assessment and training } \\
\text { tools in assessing and } \\
\text { enhancing interpersonal } \\
\text { and communication skills }\end{array}$ & $\begin{array}{c}\text { Prospective } \\
\text { control-group study }\end{array}$ & 21 residents & $-/-$ & ESCI & $\begin{array}{l}\text { EI is a necessary skill in } \\
\text { today's health care }\end{array}$ \\
\hline $\begin{array}{l}\text { Gough et al., 1991, } \\
\text { USA [42] }\end{array}$ & $\begin{array}{l}\text { Performance of residents in } \\
\text { anesthesiology as related to } \\
\text { measures of personality and } \\
\text { interests }\end{array}$ & $\begin{array}{l}\text { To study personality } \\
\text { variables }\end{array}$ & $\begin{array}{c}\text { Prospective } \\
\text { observational study }\end{array}$ & $\begin{array}{l}99 \text { residents in } \\
\text { anaesthesiology }\end{array}$ & $-/-$ & $\begin{array}{l}\text { - } \quad \text { CPI } \\
\\
\\
\text { SII }\end{array}$ & $\begin{array}{l}\text { Empathic sensing by } \\
\text { the anaesthesiologist } \\
\text { function positively }\end{array}$ \\
\hline $\begin{array}{l}\text { LePine et al., 2000, } \\
\text { USA [43] }\end{array}$ & $\begin{array}{l}\text { Adaptability to changing task } \\
\text { contexts: effects of general } \\
\text { cognitive ability, } \\
\text { conscientiousness, and } \\
\text { openness to experience }\end{array}$ & $\begin{array}{l}\text { To examine the extent to } \\
\text { which cognitive ability, } \\
\text { conscientiousness, and } \\
\text { openness to experience } \\
\text { predict decision-making } \\
\text { performance }\end{array}$ & $\begin{array}{c}\text { Prospective } \\
\text { observational study }\end{array}$ & $\begin{array}{c}73 \\
\text { undergraduates }\end{array}$ & $-/-$ & $\begin{array}{l}\text { BFIDecision making } \\
\text { performance }\end{array}$ & $\begin{array}{l}\text { Unexpected low } \\
\text { conscientiousness made } \\
\text { better decisions }\end{array}$ \\
\hline $\begin{array}{l}\text { Shankar et al., 2013, } \\
\text { Caribbean [44] }\end{array}$ & $\begin{array}{l}\text { Student attitude towards } \\
\text { communication skills learning } \\
\text { in a Caribbean Medical } \\
\text { School. }\end{array}$ & $\begin{array}{l}\text { to establish the attitude of } \\
\text { students towards to } \\
\text { communication skils }\end{array}$ & $\begin{array}{c}\text { Prospective } \\
\text { observational study }\end{array}$ & $\begin{array}{l}73 \text { undergraduate } \\
\text { medical students }\end{array}$ & $20-25$ years $/ 47.1 \% \mathrm{M}$ & CSAS & $\begin{array}{c}\text { Students overall had a } \\
\text { positive attitude } \\
\text { towards communication } \\
\text { skills }\end{array}$ \\
\hline $\begin{array}{l}\text { Akbarilakeh et al., } \\
\text { 2020, Iran [45] }\end{array}$ & $\begin{array}{l}\text { Predicating attitude toward } \\
\text { learning communication skills } \\
\text { in medical students of Shahid } \\
\text { Beheshti University. }\end{array}$ & $\begin{array}{l}\text { To investigate the attitude } \\
\text { toward learning } \\
\text { communication skills based } \\
\text { on the personality traits of } \\
\text { medical students }\end{array}$ & $\begin{array}{l}\text { Prospective } \\
\text { correlational study }\end{array}$ & $\begin{array}{l}234 \text { medical } \\
\text { students }\end{array}$ & $-/ 65 \% \mathrm{~F}$ & $\begin{array}{l}\text { - CSAS } \\
\text { Zuckerman-Kuhlman } \\
\text { personality } \\
\text { questionnaire contains } 5 \\
\text { personality dimensions }\end{array}$ & $\begin{array}{l}\text { The dimension of } \\
\text { demographic } \\
\text { characteristics are } \\
\text { effective in improving } \\
\text { the communication } \\
\text { skills of medical } \\
\text { students }\end{array}$ \\
\hline $\begin{array}{l}\text { Gheirati et al., 2016, } \\
\text { Iran [46] }\end{array}$ & $\begin{array}{l}\text { Relationship between } \\
\text { communication skills and } \\
\text { mental health among the } \\
\text { students of Mashhad } \\
\text { University Of Medical } \\
\text { Sciences }\end{array}$ & $\begin{array}{l}\text { To determine associations } \\
\text { between communication } \\
\text { skills and mental health }\end{array}$ & $\begin{array}{l}\text { Prospective } \\
\text { cross-sectional } \\
\text { analytical study }\end{array}$ & $\begin{array}{l}210 \text { medical } \\
\text { students }\end{array}$ & $-/-$ & $\begin{array}{l}\text { - Communication } \\
\text { Skills Question- } \\
\text { naires } \\
\text { General Health } \\
\text { Questionnaires }\end{array}$ & $\begin{array}{l}\text { To promote the mental } \\
\text { health of the students, } \\
\text { it is recommended to } \\
\text { conduct psychological } \\
\text { assessments of the } \\
\text { students }\end{array}$ \\
\hline $\begin{array}{l}\text { Nami et al., 2014, } \\
\text { Iran [47] }\end{array}$ & $\begin{array}{l}\text { The Role of students } \\
\text { personality traits on students } \\
\text { learning style in university of } \\
\text { medical sciences }\end{array}$ & $\begin{array}{l}\text { To investigate the } \\
\text { relationship between } \\
\text { personality traits and Kolb } \\
\text { learning style }\end{array}$ & $\begin{array}{c}\text { Prospective } \\
\text { observational study } \\
\text { (MANOVA test) }\end{array}$ & $\begin{array}{l}300 \text { medical } \\
\text { students }\end{array}$ & $-/-$ & $\begin{array}{l}\text { Neo-FFI } \\
\begin{array}{c}\text { Kolb learning style } \\
\text { questionnaire }\end{array}\end{array}$ & $\begin{array}{l}\text { Significant relationships } \\
\text { among the components } \\
\text { of personality traits and } \\
\text { their learning style }\end{array}$ \\
\hline
\end{tabular}


Table 1. Cont.

\begin{tabular}{|c|c|c|c|c|c|c|c|}
\hline $\begin{array}{l}\text { Author, Year, and } \\
\text { Country }\end{array}$ & Title of the Study & Aim & Study Type & Sample & $\begin{array}{c}\text { Median Age or Mean } \\
\text { (Years)/Gender (\%) }\end{array}$ & Methods/Scales & Observations \\
\hline $\begin{array}{l}\text { Molinuevo et al., } \\
\text { 2013, Spain [48] }\end{array}$ & $\begin{array}{l}\text { Does personality predict } \\
\text { medical students' attitudes to } \\
\text { learning communication } \\
\text { skills? }\end{array}$ & $\begin{array}{c}\text { To determine whether } \\
\text { personality is related to } \\
\text { medical students' attitudes } \\
\text { towards learning } \\
\text { communication skills and } \\
\text { self-ratings on } \\
\text { communication skills }\end{array}$ & $\begin{array}{c}\text { Prospective } \\
\text { control-group study }\end{array}$ & $\begin{array}{l}1031 \text { medical } \\
\text { students (divided } \\
\text { in } 2 \text { groups: } 524 \\
\text { 1st-year students } \\
\text { and } 507 \text { 2nd-year } \\
\text { students) }\end{array}$ & $\begin{array}{c}18.89 \pm 3.21 \\
\text { years-1st-year } \\
\text { students } / 66 \% \mathrm{~F} \\
20.11 \pm 3.10 \\
\text { years-2nd-year } \\
\text { students } / 70 \% \mathrm{~F}\end{array}$ & $\begin{array}{ll}\bullet \quad & \text { CSAS } \\
& \text { EPQ } \\
& \text { ZKPQ }\end{array}$ & $\begin{array}{l}\text { Personality traits are } \\
\text { useful for better student } \\
\text { career guidance and } \\
\text { counselling }\end{array}$ \\
\hline $\begin{array}{l}\text { Zare-Alamshiri } \\
\text { et al., 2017, Iran [49] }\end{array}$ & $\begin{array}{l}\text { Prediction of communication } \\
\text { skills based on psycho-social } \\
\text { class atmosphere and social } \\
\text { anxiety of high school } \\
\text { students }\end{array}$ & $\begin{array}{l}\text { To predict the psychological } \\
\text { atmosphere of social } \\
\text { communication skills and } \\
\text { social anxiety in high school } \\
\text { students }\end{array}$ & $\begin{array}{c}\text { Prospective } \\
\text { observational study }\end{array}$ & $\begin{array}{l}210 \text { high school } \\
\text { students }\end{array}$ & $-/-$ & $\begin{array}{l}\text { - } \quad \text { Social anxiety } \\
\text { scale } \\
\text { CSAS } \\
\text { classroom psychosocial } \\
\text { climate scale }\end{array}$ & $\begin{array}{l}\text { Psychosocial classroom } \\
\text { atmosphere can predict } \\
\text { communication skills } \\
\text { and social anxiety }\end{array}$ \\
\hline $\begin{array}{l}\text { O'Tuathaigh et al., } \\
\text { 2019, Ireland [50] }\end{array}$ & $\begin{array}{l}\text { Medical students' empathy } \\
\text { and attitudes towards } \\
\text { professionalism: relationship } \\
\text { with personality, specialty } \\
\text { preference and medical } \\
\text { programme }\end{array}$ & $\begin{array}{l}\text { To examine how empathy, } \\
\text { personality, and background } \\
\text { factors might impact } \\
\text { students' attitudes towards } \\
\text { professionalism in medicine }\end{array}$ & $\begin{array}{c}\text { Prospective } \\
\text { cross-sectional } \\
\text { questionnaire-based } \\
\text { study }\end{array}$ & $\begin{array}{l}241 \text { medical } \\
\text { students }\end{array}$ & $\begin{array}{c}18-22(46.6 \%), 23-27 \\
(43.3 \%), 28-32(8.8 \%), \\
33-37(0.8 \%), 38-42 \\
(0.5 \%) / 49.2 \% \mathrm{~F}\end{array}$ & $\begin{array}{l}\text { JSE } \\
\text { NEO-FFI } \\
\text { Attitudes towards } \\
\text { professionalism scale }\end{array}$ & $\begin{array}{l}\text { Empathy and } \\
\text { personality factors may } \\
\text { act as determinants of } \\
\text { students' attitudes }\end{array}$ \\
\hline $\begin{array}{l}\text { Sims et al., 2017, } \\
\text { United Kingdom } \\
\text { [51] }\end{array}$ & $\begin{array}{l}\text { Do the big-five personality } \\
\text { traits predict empathic } \\
\text { listening and assertive } \\
\text { communication? }\end{array}$ & $\begin{array}{l}\text { To investigate whether the } \\
\text { Big Five had predictive } \\
\text { influences on } \\
\text { communication } \\
\text { competences of } \\
\text { active-empathic listening } \\
\text { (AEL) and assertiveness }\end{array}$ & $\begin{array}{c}\text { Prospective } \\
\text { observational study }\end{array}$ & 245 adults & $\begin{array}{c}<25 \text { years } 59 ; \\
26-35 \text { years: } 42 ; 36-45 \\
\text { years: } 50 ; 46-55 \text { years: } 64 ; \\
>56 \text { years: } 28 / 75.9 \% \mathrm{~F}\end{array}$ & $\begin{array}{l}\bullet \quad \text { AEL } \\
\quad \text { IPIP } \\
\quad \text { RAS }\end{array}$ & $\begin{array}{l}\text { Agreeableness and } \\
\text { Openness uniquely } \\
\text { predicted AEL } \\
\text { Extraversion had the } \\
\text { biggest influence on } \\
\text { assertiveness }\end{array}$ \\
\hline $\begin{array}{c}\text { Kuntze etl., 2016, } \\
\text { Holland [52] }\end{array}$ & $\begin{array}{l}\text { Big five personality traits and } \\
\text { assertiveness do not affect } \\
\text { mastery of communication } \\
\text { skills. }\end{array}$ & $\begin{array}{l}\text { To investigate whether the } \\
\text { big-five personality factors } \\
\text { and assertiveness predict } \\
\text { mastery of communication } \\
\text { skills }\end{array}$ & $\begin{array}{c}\text { Prospective } \\
\text { observational study }\end{array}$ & $\begin{array}{l}143 \text { bachelor } \\
\text { students of a } \\
\text { psychology } \\
\text { curriculum }\end{array}$ & $19 / 83 \% \mathrm{~F}$ & $\begin{array}{ll}- & \text { FFPI } \\
& \text { SIB } \\
& \text { CSPT }\end{array}$ & $\begin{array}{l}\text { Trainees can become } \\
\text { professional } \\
\text { communicators, } \\
\text { regardless of their } \\
\text { scores on these } \\
\text { personality factors }\end{array}$ \\
\hline $\begin{array}{c}\text { Franco et al., 2020, } \\
\text { Brazilia [53] }\end{array}$ & $\begin{array}{l}\text { The assessment of personality } \\
\text { traits and its association with } \\
\text { learning communication skills }\end{array}$ & $\begin{array}{c}\text { To investigate the } \\
\text { association between } \\
\text { personality traits and } \\
\text { attitudes toward learning } \\
\text { communication skills in } \\
\text { undergraduate medical } \\
\text { students }\end{array}$ & $\begin{array}{c}\text { Prospective } \\
\text { observational study }\end{array}$ & 204 students & $-/-$ & $\begin{array}{l}\text { - CSAS } \\
\text { BFMM }\end{array}$ & $\begin{array}{c}\text { Elation between } \\
\text { agreeableness, } \\
\text { extraversion and } \\
\text { openness to experience } \\
\text { with attitudes on } \\
\text { communication skills in } \\
\text { students }\end{array}$ \\
\hline
\end{tabular}


Table 1. Cont.

\begin{tabular}{|c|c|c|c|c|c|c|c|}
\hline $\begin{array}{l}\text { Author, Year, and } \\
\text { Country }\end{array}$ & Title of the Study & Aim & Study Type & Sample & $\begin{array}{c}\text { Median Age or Mean } \\
\text { (Years)/Gender (\%) }\end{array}$ & Methods/Scales & Observations \\
\hline $\begin{array}{l}\text { Vermetten et al., } \\
\text { 2001, The } \\
\text { Netherlands [54] }\end{array}$ & $\begin{array}{l}\text { The Role of personality traits } \\
\text { and goal orientations in } \\
\text { strategy use }\end{array}$ & $\begin{array}{l}\text { To contribute to the } \\
\text { development of an } \\
\text { integrated theory on } \\
\text { individual learning } \\
\text { differences }\end{array}$ & $\begin{array}{c}\text { Prospective } \\
\text { observational study }\end{array}$ & 310 students & $21.5 / 75 \% \mathrm{~F}$ & $\begin{array}{l}\text { - } \quad \text { ILS } \\
\text { - } \quad \text { Goal orientation } \\
\text { scale } \\
\text { SITIQ } \\
\text { Big Five personality } \\
\quad \text { factors }\end{array}$ & $\begin{array}{l}\text { Individual differences } \\
\text { in learning consist of } \\
\text { and help explain } \\
\text { regularities in learning } \\
\text { behaviour }\end{array}$ \\
\hline $\begin{array}{l}\text { Holen et al., 2015, } \\
\text { Norway [55] }\end{array}$ & $\begin{array}{l}\text { Medical students' preferences } \\
\text { for problem-based learning in } \\
\text { relation to culture and } \\
\text { personality: a multicultural } \\
\text { study }\end{array}$ & $\begin{array}{l}\text { To explore positive and } \\
\text { negative preferences } \\
\text { towards PBL in relation to } \\
\text { personality traits and } \\
\text { sociocultural context }\end{array}$ & $\begin{array}{c}\text { Prospective } \\
\text { cross-sectional survey }\end{array}$ & $\begin{array}{c}449 \text { medical } \\
\text { students (123 } \\
\text { from Nepal, } 229 \\
\text { from Norway and } \\
97 \text { from the USA) }\end{array}$ & $\begin{array}{c}21.4 \text { Nepal } / 34.9 \% \text { F } \\
22.1 \text { Norway } / 55.28 \% \text { F } \\
24.1 \text { USA } 46.10 \% \text { F }\end{array}$ & PBL & $\begin{array}{l}\text { Preferences related to } \\
\text { PBL were significantly } \\
\text { and independently } \\
\text { determined by } \\
\text { personality traits and } \\
\text { culture }\end{array}$ \\
\hline $\begin{array}{l}\text { Tsou et al., 2013, } \\
\text { Taiwan [56] }\end{array}$ & $\begin{array}{l}\text { Using personal qualities } \\
\text { assessment to measure the } \\
\text { moral orientation and } \\
\text { personal qualities of medical } \\
\text { students in a non-western } \\
\text { culture }\end{array}$ & $\begin{array}{l}\text { To select candidates with } \\
\text { appropriate personal } \\
\text { qualities for medical school }\end{array}$ & $\begin{array}{c}\text { Prospective } \\
\text { observational study }\end{array}$ & $\begin{array}{l}746 \text { medical } \\
\text { students }\end{array}$ & $\begin{array}{l}20.3 \pm 2.2 / \\
65.8 \% \mathrm{M}\end{array}$ & $\begin{array}{l}\text { Mojac and NACE } \\
\text { scale of PQA } \\
\text { Big Five" personality } \\
\text { traits }\end{array}$ & $\begin{array}{c}\text { Significant relationships } \\
\text { were observed between } \\
\text { test components and } \\
\text { between the NACE and } \\
\text { Big } 5\end{array}$ \\
\hline
\end{tabular}

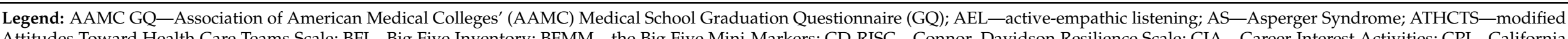

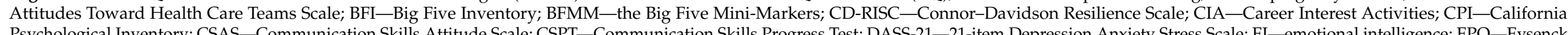

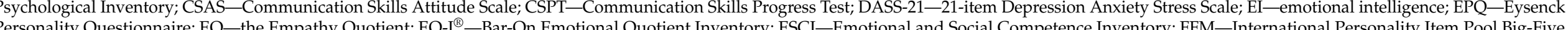

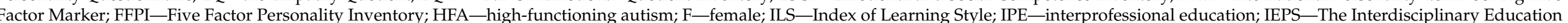

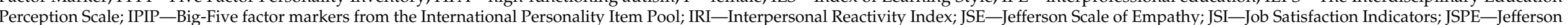

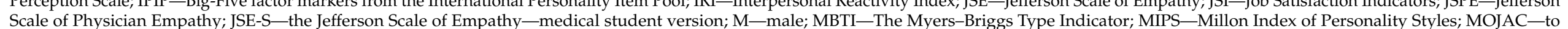

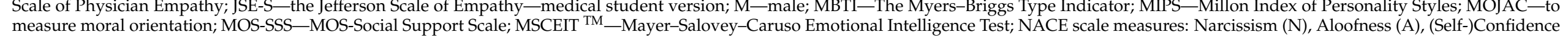

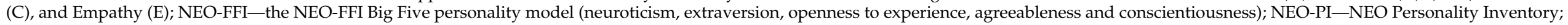

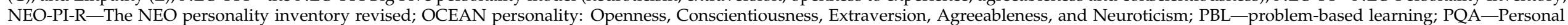

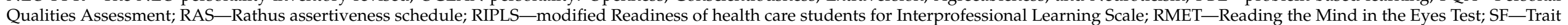

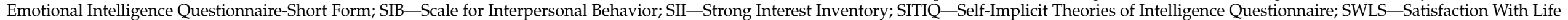

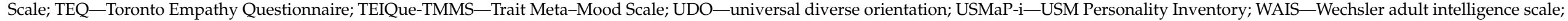
ZKPQ—Zuckerman-Kuhlman Personality Questionnaire; ZKPQ-S—Zuckerman-Kuhlman personality questionnaire-short form. 


\section{Personality Traits in Medical Students-The Core of the Doctor-Patient Relationship}

As mentioned above, each individual has their own way of thinking, behaving and expressing feelings or emotions, characteristics that define an individual's personality. Moreover, personality is known as one of the most crucial aspects of human life [11]. It is also known to influence all human behaviours in both personal and social life, but it might also trigger important conflicts with a person's surroundings in the setting of incompatible traits and characteristics [12]. Two famous personality theorists, Zweig and Webster, emphasise that the differences in an individual's personality represent probably the most important factor that influences a human's motivation in learning, performance, and behaviour, considering that all the controversies in areas such as thinking, intelligence, perception, emotions, learning, and motivation are based on this issue [11]. Taking into account that effective doctor-patient communication represents the cornerstone of patientand family-centred care, medical training should focus on improving personality traits that influence this communication. Nevertheless, this fact cannot be achieved without a proper assessment of personality traits.

One of the most frequently used tools for assessing personality is represented by the 'big-five model' and its derivatives, e.g., Personality Inventory NEO-PI-R [13]. The big-five model or the five-factor model has been used for assessing intercultural differences among different countries, defining the underlying qualities of personality traits $[57,58]$. Thus, the big-five model is based on the assessment of five dimensions of personality and its related facets, six for each dimension: Openness, facets-fantasy, aesthetics, feelings, actions, ideas, values; Extraversion, facets-warmth, gregariousness, assertiveness, activity, excitement-seeking, positive emotions; Conscientiousness, facets-competence, order, dutifulness, achievement striving, self-discipline, deliberation; Agreeability, facets-trust, straightforwardness, altruism, compliance, modesty, tender-mindedness; Neuroticism, facets-anxiety, angry hostility, depression, self-consciousness, impulsiveness, vulnerability $[14,59]$. Each of these dimensions is related to specific personality aspects and defines particular personality subdimensions. Thus, Openness is related to an individual's intellectual interests, culture and openness to experiences [15]. Extraversion is a reliable indicator of energy, enthusiasm, sociability, orientation towards others and task perseverance [60,61]. Conscientiousness is another personality dimension that implies prudence, responsibility, moral integrity, the need for structure and order, as well as perseverance in action [60]. Interpersonal relationships represent a fair reflection of agreeability, which is related to altruism, goodwill, gentleness, direct behaviour and trust in people [60]. Emotional Stability or Neuroticism is associated with depression, impulsivity, anxiety, vulnerability and the tendency to worry [60]. This model was assessed in multiple general studies, but unfortunately, its utility in medical students is far from being properly assessed. Taking into account the great impact of their personality on their professional skills and performance, a proper understanding of their personality would be of great benefit during medical school.

Professional satisfaction is a strong hallmark of wellbeing during adulthood. In terms of the medical profession, this is choosing a specialty that matches best with an individual's personality traits. Multiple factors were identified as essential contributors for medical specialty choice such as personality, gender, personal interest, economic status, clinical experience during clerkship, mentoring from professor, expected income, lifestyle, family or public media influence [16-18]. Thus, extrinsic factors display a greater influence on a student's decision when choosing their specialty. Nevertheless, personality remains a major determinant in specialty decision making. Taking into account the major impact of this choice on public medical services and policy on medical education, each career counsellor or professor that mentors medical students should focus more on finding an effective method to assess their personality [62]. Moreover, a proper assessment of personality traits might predict performance in carrying out tasks entailed by each medical specialty [6]. Thus, we are definitely entitled to say that 'one must be born' for a certain medical profession in order to achieve the desired performance. A recent study performed 
on medical students aimed to identify the relationship between specialty choice and personality traits, and concluded that students with more Agreeableness preferred clinical medicine instead of basic medicine [6]. The authors also underlined that students with an increased level of Openness intended to choose medical departments [6]. Openness was also proved to be directly related to satisfaction and personal interest when choosing the medical specialty [6]. Unsurprisingly, age was shown to influence certain personality traits such as Conscientiousness and Agreeableness, which seem to be higher in older students [6]. Another study that compared surgical residents with the general population in terms of personality traits pointed out that surgeons scored significantly higher for Extraversion, Conscientiousness and Openness [19]. According to the study of Hoffman et al., who compared the personalities of surgical residents, nonsurgical residents and medical students, surgical residents scored better for Conscientiousness and Extraversion, but worse for Openness than medical students [20]. Thus, previous studies emphasised the fact that surgeons possess a particular personality distinct from that of trainees in other medical specialties or the general population $[19,63]$, suggesting there is an innate character of their personality that enables them to perform this specialty. Nevertheless, the question of whether surgeons are born or made is rather challenging. Thus, a recent study performed on surgical trainees and medical students who intend to pursue a surgical specialty proved that the two groups display multiple similarities regarding both personality traits and learning styles [21], indicating that most likely an innate personality is required for this profession and these traits are neither learnt or developed during surgical training. Both groups included in this study scored high for Conscientiousness, Agreeableness and Openness, and low for Neuroticism [21]. These findings suggest that trainees adjust their Neuroticism during residency programs and become more emotionally stable over the course of surgical training, experiencing lower anxiety and depression levels as compared to undergraduates that aim to pursue a surgical career. Moreover, the combination between increased levels of Conscientiousness, Agreeableness and Openness and lower levels of Neuroticism might represent the key attribute for enabling students to accept better and cope with the tasks and rigor of a stressful residency program such as surgical training. Thus, personality testing should be mandatory during medical education since it was clearly showed that personality profiling is an extremely useful tool for distinguishing between high- and low-performing residents [22]. Taking into account the crucial impact of the match between personality traits and medical career on the health care services and national economy, each university should implement a program designed for personality testing during the medical training in order to properly guide medical students in their specialty choice.

Interprofessional collaboration between healthcare providers is essential in order to achieve the best outcome for each patient and improve patient safety. Thus, the collaboration of different healthcare professionals in well-functioning teams by contributing with their specific knowledge related to each medical specialty, resulting in a holistic approach, will definitely impact both medical performance and patient satisfaction [22,64]. Interprofessional education is meant to provide students opportunities to develop, train, and learn collaboration skills and each medical university should implement this type of education as a regular training, since it is often challenging to achieve good interprofessional teamwork [64]. It is true that awareness regarding the need for interprofessional education during medical school has increased considerably during the last few years, but students' attitudes towards this type of training remain an issue since they seem to be influenced by multiple factors such as gender, learning styles or personality traits [8,23-27]. Thus, once more, we emphasise that a thorough understanding of personality traits in medical students could considerably improve their attitudes towards interprofessional education and collaboration by providing teachers the opportunity to design proper teaching methods adjusted to medical students' personality traits and their preferences for different learning styles. 
Medical empathy, defined as the ability to understand a patient's experiences, concerns, and perspectives and to express this understanding in their favour [24], is another essential concept that contributes to effective patient-physician communication, improving patient trust, compliance, and satisfaction in order to achieve the best outcome in clinical practice [28]. Thus, it was fairly stated that the physician's performance, clinical skills, and biomedical knowledge are strongly influenced by empathy and emotional abilities, which, in turn, are shaped by personality [29]. In terms of personality traits, Openness and Agreeableness were proven to be positively correlated with empathy [30]. Moreover, it seems that empathy is also positively correlated with two other personality dimensions, i.e., Conscientiousness and Extraversion [1]. Nevertheless, it is also true that increased empathy might result in emotional fatigue, increased risk of stress, and subsequent 'burn out' [65]. Self-assessment and understanding of personality traits could considerably decrease the previously mentioned risks by approaching suitable training strategies in order to improve unfavourable traits. The main daily goal for each physician is to provide the best of care for their patients and, therefore, the relationship between empathy, emotional intelligence, and personality was found to be the main factor that contributes to successful achievement of this goal. Multiple studies proved that emotional intelligence is associated with increased empathy, improved patient-physician relationship, teamwork, communication skills, stress management, and leadership resulting in higher academic performance $[31,32,65]$. The relationship between emotional intelligence and personality is further emphasised by the fact that the first one includes the ability to understand, perceive, and manage self-emotions, but also to perceive and understand the emotions of other humans [66]. In spite of their inherited and innate pattern, studies proved that emotional intelligence and empathy can be improved through proper education, implying an urgent need to implement communication training in medical students, since they seem to have a positive influence on both emotional intelligence and empathy $[33,34,67]$. Thus, an effective program designed for enhancing emotional intelligence and empathy in medical students should definitely take into account students' personality $[35,67,68]$. The implementation of such a program during medical school is crucial, since it was proved that empathy declines during academic medical school training [36]. In addition, the decreasing pattern of empathy continues after they begin to practice their profession, since a doctor's life is best characterised by resulting in an impairment of personality traits and devotion to patients. Therefore, programs for enhancing empathy would be of major benefit for improving professional experiences and performance.

A close interplay between personality traits, empathy, and interprofessional collaboration was emphasised by recent studies, which proved that empathy is positively associated with collaborative interprofessional work in postgraduates who are at the beginning of their specialisation [37], being well-documented that both empathy and interprofessional work are influenced by personality traits. Moreover, it was underlined that general empathy might be predicted by different personality styles, such as styles of protection, sensation, and accommodation [38]. In terms of interprofessional collaboration and communication skills, it was noticed that individuals that display a greater degree of empathy find it easier to accept failures in their life, being more optimistic regarding the future, motivated to satisfy others first, and trying to change their environment to fulfil their goals [38]. According to Zaki et al., empathy is a motivated phenomenon implying a close interaction between social desirability and intrinsic motivation [69]. Thus, a student's self-efficacy is moderated mainly by two motivational factors, i.e., value of the goal for which they work and the expectation to achieve that goal [39]. Nevertheless, intrinsic motivation and self-efficacy are not enough to achieve the goal in medicine if not supported by empathy. The complexity of practicing and understanding empathy in clinical situations requires a solid basis provided by intense training in terms of personal development, relationship and system maintenance, chance, and goal progress [40].

Emotional intelligence is another crucial ability for medical students, since it represents a measure of emotional awareness and the capacity to respond to one's own and 
others' emotions. Thus, Mayer and Salovey stated that emotional intelligence ability implies not only the ability to perceive and integrate emotion, but also to understand and regulate emotion in order to favour personal growth [29,70]. The Accreditation Council for Graduate Medical Education defined emotional intelligence competency as a set of assessment methods for interpersonal and communication skills, patient care, and professional behaviours in residents [71]. Thus, 12 abilities were related to emotional intelligence competency: emotional self-awareness, emotional self-control, achievement orientation, adaptability, empathy, positive outlook, coach and mentor, organisational awareness, influence, inspirational leadership, teamwork, and conflict management, which represent, along with empathy, the most important traits of $21^{\text {st }}$ century physicians [41]. An increased level of emotional intelligence was shown to positively influence doctor-patient relationship, teamwork, communication skills, level of empathy, organisation commitment, as well as stress management $[31,32,65,72]$. Emotional intelligence and empathy synergistically contribute to increasing empathic ability [29]. One of the most important deficiencies in medical students is a lack of confidence and resources to implement empathic behaviour, despite them fully acknowledging and understanding its value [29], emphasising once more the need for proper dedicated training during medical school. Therefore, medical education should focus not only on improving empathy and empathic behaviour in medical students, but also promoting and teaching methods for its effective implementation in residents and young physicians. Emotional intelligence is definitely influenced by personality. A recent study performed on US medical students, which focused on assessing emotional intelligence, revealed a strong positive association with Extraversion, a moderate positive one with Conscientiousness, Agreeability and Openness, as well as a weak negative association with Neuroticism [35]. Moreover, Conscientiousness and Openness were found to be reliable predictors for medical students' competencies in clinical contexts [42]; Openness and Agreeability promote the development of patient-physician relationships [43], as well as training proficiency [73]; and Conscientiousness was identified as the most important trait required for effective task completion [74]. Considering the complex aforementioned findings, emotional intelligence has an incontestable influence on patient-physician relationships, medical students' and physicians' communication skills, professional performance, and proficiency, creating important bridges with empathy and interprofessional collaboration skills under the close guidance of personality traits.

Clinical communication skills represent the key component in medical students in order to achieve the best patient care, and they are certainly related and/or influenced by empathy, interprofessional collaboration skills, emotional intelligence, and especially personality traits. Nevertheless, effective communication between physician and patient/caregivers represents the 'cornerstone' of care by improving the patient's outcome. Good communication skills improve not only the physical but also the mental health of patients, increasing their compliance and satisfaction regarding the provided care [44]. The physician's ability to communicate and their knowledge represent two major factors that equally contribute to the patient's outcome [45]. In addition, personality traits were proven to also affect attitudes regarding communication skills training, although these skills are currently considered a crucial attribute for all people working in primary health care systems [46]. Medical errors, a continuous cause of death displaying an increasing pattern worldwide, seem to be mainly related to a major lack of communication skills [47]. It is indeed a real fact that the current trends in medicine focus on the acquisition of proper communication skills in medical students and not only on enhancing the theoretical aspects of each medical field, underlining that these skills should be considered when determining physicians' competence [11]. Thus, medical educators should pay close attention and combine students' personality and the ability to learn the communication skills in order to develop effective communication skills training to reduce medical errors in the long run [45]. Based on these findings, we hypothesise that physicians' appropriate and effective ability to communicate might result in a considerable reduction in medical errors and subsequent deaths. Medical educators should also focus on increasing students' awareness 
regarding the importance of learning communication skills, since it was underlined that they find no merit in improving these skills because they do not acknowledge them as an important aspect of medical and practical education [11]. This fact is rather concerning considering the effectiveness of students' attitudes and satisfaction with learning in motivating and promoting knowledge quality [45]. Recent studies focusing on medical students' personality traits and their attitude towards learning communication skills concluded that anxiety was associated with a negative perspective regarding communication skills training during medical school $[48,49]$, while students with increased sociability levels were found to have a positive attitude towards this training $[45,48]$. Moreover, Agreeableness, Extraversion, and Conscientiousness were positively correlated with medical students' attitudes regarding the interaction with patients, underlining that these personality dimensions might also be related to students' attitudes in terms of communication [50]. Contrariwise, aggression/hostility represents the main characteristic of antisocial and bold behaviours, and it was associated with a negative attitude towards learning communication skills, since individuals with this type of personality express no interest in communicating with others by lacking this desired attitude [45].

The importance of clinically focused skills and behaviours in medical education has been acknowledged by both the Association of American Medical Colleges and the European Board of Internal Medicine for over a decade [75,76]. The competencies related to clinical communication skills and to clinical practice include trust building; respect; compassion; altruism; empathy; understanding patient's spirituality, beliefs and meaning; professionalism; information sharing and mutual decision making; relationship building; cultural sensitivity; responsiveness to distress; appreciation of psychological factors; self-awareness; ethical issues; and acting with honesty [75,76]. These competencies were related to personality dimensions in several studies, but the results remain contradictory depending on the studied populations. Therefore, Sims et al. assessed the relationship between personality traits and communication skills in adults and showed that Agreeableness and Openness might predict assertiveness and empathic listening in communication [51]. Contrariwise, a study performed on psychology students revealed no significant correlation between the same neither before nor after communication training [52]. Studies performed on medical students remain scarce. Nevertheless, Agreeableness, Openness, and Extraversion were found to be the most important factors that positively influence students attitude towards communication and learning, enhancing a better connection between the patient and physician [53]. Moreover, each of these dimensions were proven to have a major contribution to achieving the ultimate goal of improving students' attitudes towards communication in different ways: Agreeableness enables students to initiate a relationship with patients easier by providing the tendency to be friendly [54], Openness promotes the better acceptance of adversity and willingness to change [55], while Extraversion favours energy to social interaction better expressing the inner disposition [56]. Based on all these evidence-based statements, medical teachers should focus more on assessing students' personality traits and implement their teaching activities tailored accordingly or even support the development of personality dimensions that favour an effective doctor-patient communication.

\section{Conclusions}

The relationship between physician and patient represents the core of medicine and all efforts should focus on improving this relationship. A thorough assessment of medical students' personality traits is crucial for educators in order to acknowledge the main deficiencies in their students and to design effective tailored communication and clinical practice training for improving and educating these deficiencies. Professional satisfaction is definitely influenced by career choice, which should be properly guided after an individual assessment of personality traits. Interprofessional collaboration and teamwork are mandatory for patient safety and medical students should be made aware of its importance even before graduating. Emotional intelligence and empathy act as partners for improving em- 
pathic ability in order to reach the best doctor-patient relationship. Empathy is positively influenced by being positively correlated with Openness, Agreeableness, Conscientiousness, and Extraversion, and thus, training should focus on improving these components in medical students. The ultimate goal of the medical profession is to achieve the best outcome for each patient. Empathy, emotional intelligence, and clinical communication skills are essential for building an effective doctor-patient relationship, while interprofessional collaboration and teamwork are mandatory for assuring the best of care in practice, altogether being closely governed by personality traits and crucial for providing the best outcome for the patients. Taking into account students' personality traits, courses should aim to improve empathy, clinical communication skills, interprofessional collaboration, teamwork, and practical competencies for the patients' best outcomes. Thus, proper training should begin in medical students and should never end.

Author Contributions: N.S.-Conceptualisation, Data curation, Investigation, Methodology, Project administration, Visualisation, Writing-original draft, Writing-review and editing, C.O.M.Conceptualisation, Project administration, Visualisation, Supervision, Writing —original draft, Writingreview and editing, L.E.M.-Conceptualisation, Visualisation, Writing-original draft, Writingreview and editing. All authors approved the final manuscript as submitted and agree to be accountable for all aspects of the work. All authors have read and agreed to the published version of the manuscript.

Funding: This research received no external funding.

Institutional Review Board Statement: The study was conducted according to the guidelines of the Declaration of Helsinki, and approved by the Ethics Committee of the University of Medicine, Pharmacy, Sciences and Technology from Târgu Mureș (No. 1157/20.10.2020).

Informed Consent Statement: Informed consent was obtained from all subjects involved in the study.

Conflicts of Interest: The authors declare no conflict of interest.

\section{References}

1. Guilera, T.; Batalla, I.; Forné, C.; Soler-González, J. Empathy and Big Five Personality Model in Medical Students and Its Relationship to Gender and Specialty Preference: A Cross-Sectional Study. BMC Med. Educ. 2019, 19, 57. [CrossRef]

2. Baron-Cohen, S.; Wheelwright, S. The Empathy Quotient: An Investigation of Adults with Asperger Syndrome or High Functioning Autism, and Normal Sex Differences. J. Autism Dev. Disord. 2004, 34, 163-175. [CrossRef] [PubMed]

3. Sulzer, S.H.; Feinstein, N.W.; Wendland, C.L. Assessing Empathy Development in Medical Education: A Systematic Review. Med. Educ. 2016, 50, 300-310. [CrossRef] [PubMed]

4. Hojat, M.; Gonnella, J.S. Eleven Years of Data on the Jefferson Scale of Empathy-Medical Student Version (JSE-S): Proxy Norm Data and Tentative Cutoff Scores. Med. Princ. Pract. Int. J. Kuwait Univ. Health Sci. Cent. 2015, 24, 344-350. [CrossRef]

5. Yusoff, M. Which Personality Traits Have Favourable Impact on Psychological Health during Stressful Condition? Educ. Med. J. 2018, 10, 35-42. [CrossRef]

6. Kwon, O.Y.; Park, S.Y. Specialty Choice Preference of Medical Students According to Personality Traits by Five-Factor Model. Korean J. Med. Educ. 2016, 28, 95-102. [CrossRef]

7. McCrae, R.R.; Costa, P.T. Reinterpreting the Myers-Briggs Type Indicator from the Perspective of the Five-Factor Model of Personality. J. Personal. 1989, 57, 17-40. [CrossRef] [PubMed]

8. Olsson, C.; Lachmann, H.; Kalén, S.; Ponzer, S.; Mellstrand Navarro, C. Personality and Learning Styles in Relation to Attitudes towards Interprofessional Education: A Cross-Sectional Study on Undergraduate Medical Students during Their Clinical Courses. BMC Med. Educ. 2020, 20, 398. [CrossRef] [PubMed]

9. Derish, P.A.; Annesley, T.M. How to Write a Rave Review. Clin. Chem. 2011, 57, 388-391. [CrossRef]

10. Du Prel, J.-B.; Röhrig, B.; Blettner, M. Critical Appraisal of Scientific Articles: Part 1 of a Series on Evaluation of Scientific Publications. Dtsch. Arztebl. Int. 2009, 106, 100-105. [CrossRef]

11. Tamannaeifar, M.; Mansourinik, A. The Relationship between Personality Characteristics, Social Support and Life Satisfaction with University Students' Academic Performance. Q. J. Res. Plan. High. Educ. 2014, 20, 149-166.

12. Nasri, S.; Heydari Bafghi, R.; Jararh, J. Personality Characteristics, Irrational Beliefs, and Communication Skills as Predictors School Counselors' Job Performance. Biannu. J. Appl. Couns. 2017, 7, 27-46. [CrossRef]

13. Vassend, O.; Skrondal, A. The NEO Personality Inventory Revised (NEO-PI-R): Exploring the Measurement Structure and Variants of the Five-Factor Model. Personal. Individ. Differ. 2011, 50, 1300-1304. [CrossRef] 
14. Lievens, F.; Coetsier, P.; De Fruyt, F.; De Maeseneer, J. Medical Students' Personality Characteristics and Academic Performance: A Five-Factor Model Perspective. Med. Educ. 2002, 36, 1050-1056. [CrossRef] [PubMed]

15. Han, S.; Pistole, M.C. Big Five Personality Factors and Facets as Predictors of Openness to Diversity. J. Psychol. 2017, 151, 752-766. [CrossRef] [PubMed]

16. Borges, N.J.; Stratton, T.D.; Wagner, P.J.; Elam, C.L. Emotional Intelligence and Medical Specialty Choice: Findings from Three Empirical Studies. Med. Educ. 2009, 43, 565-572. [CrossRef] [PubMed]

17. Hojat, M.; Zuckerman, M. Personality and Specialty Interest in Medical Students. Med. Teach. 2008, 30, 400-406. [CrossRef]

18. Lambert, E.M.; Holmboe, E.S. The Relationship between Specialty Choice and Gender of U.S. Medical Students, 1990-2003. Acad. Med. J. Assoc. Am. Med. Coll. 2005, 80, 797-802. [CrossRef]

19. McGreevy, J.; Wiebe, D. A Preliminary Measurement of the Surgical Personality. Am. J. Surg. 2002, 184, 121-125. [CrossRef]

20. Hoffman, B.M.; Coons, M.J.; Kuo, P.C. Personality Differences between Surgery Residents, Nonsurgery Residents, and Medical Students. Surgery 2010, 148, 187-193. [CrossRef]

21. Preece, R.A.; Cope, A.C. Are Surgeons Born or Made? A Comparison of Personality Traits and Learning Styles Between Surgical Trainees and Medical Students. J. Surg. Educ. 2016, 73, 768-773. [CrossRef] [PubMed]

22. Foster, K.N.; Neidert, G.P.M.; Brubaker-Rimmer, R.; Artalejo, D.; Caruso, D.M. A Psychological Profile of Surgeons and Surgical Residents. J. Surg. Educ. 2010, 67, 359-370. [CrossRef] [PubMed]

23. Hayashi, T.; Shinozaki, H.; Makino, T.; Ogawara, H.; Asakawa, Y.; Iwasaki, K.; Matsuda, T.; Abe, Y.; Tozato, F.; Koizumi, M.; et al. Changes in Attitudes toward Interprofessional Health Care Teams and Education in the First- and Third-Year Undergraduate Students. J. Interprof. Care 2012, 26, 100-107. [CrossRef]

24. Wilhelmsson, M.; Ponzer, S.; Dahlgren, L.-O.; Timpka, T.; Faresjö, T. Are Female Students in General and Nursing Students More Ready for Teamwork and Interprofessional Collaboration in Healthcare? BMC Med. Educ. 2011, 11, 15. [CrossRef]

25. Alghasham, A.A. Effect of Students' Learning Styles on Classroom Performance in Problem-Based Learning. Med. Teach. 2012, 34 (Suppl. 1), S14-S19. [CrossRef]

26. Tariq, S.; Khan, M.A.; Afzal, S.; Shahzad, S.R.; Hamza, M.; Khan, H.A.; Qamar, F.; Javed, M.; Ashraf, M.F.; Mubarak, F.; et al. Association between Academic Learning Strategies and Annual Examination Results among Medical Students of King Edward Medical University. Ann. King Edw. Med. Univ. 2016, 22. [CrossRef]

27. Avrech Bar, M.; Katz Leurer, M.; Warshawski, S.; Itzhaki, M. The Role of Personal Resilience and Personality Traits of Healthcare Students on Their Attitudes towards Interprofessional Collaboration. Nurse Educ. Today 2018, 61, 36-42. [CrossRef] [PubMed]

28. Hojat, M.; Spandorfer, J.; Louis, D.Z.; Gonnella, J.S. Empathic and Sympathetic Orientations toward Patient Care: Conceptualization, Measurement, and Psychometrics. Acad. Med. J. Assoc. Am. Med. Coll. 2011, 86, 989-995. [CrossRef] [PubMed]

29. Abe, K.; Niwa, M.; Fujisaki, K.; Suzuki, Y. Associations between Emotional Intelligence, Empathy and Personality in Japanese Medical Students. BMC Med. Educ. 2018, 18, 47. [CrossRef]

30. Costa, P.; Alves, R.; Neto, I.; Marvão, P.; Portela, M.; Costa, M.J. Associations between Medical Student Empathy and Personality: A Multi-Institutional Study. PLoS ONE 2014, 9, e89254. [CrossRef]

31. Chew, B.H.; Zain, A.M.; Hassan, F. Emotional Intelligence and Academic Performance in First and Final Year Medical Students: A Cross-Sectional Study. BMC Med. Educ. 2013, 13, 44. [CrossRef]

32. Aithal, A.P.; Kumar, N.; Gunasegeran, P.; Sundaram, S.M.; Rong, L.Z.; Prabhu, S.P. A Survey-Based Study of Emotional Intelligence as It Relates to Gender and Academic Performance of Medical Students. Educ. Health Abingdon Engl. 2016, 29, 255-258. [CrossRef]

33. Magalhães, E.; Costa, P.; Costa, M.J. Empathy of Medical Students and Personality: Evidence from the Five-Factor Model. Med. Teach. 2012, 34, 807-812. [CrossRef] [PubMed]

34. Hojat, M.; Axelrod, D.; Spandorfer, J.; Mangione, S. Enhancing and Sustaining Empathy in Medical Students. Med. Teach. 2013, 35, 996-1001. [CrossRef]

35. Bertram, K.; Randazzo, J.; Alabi, N.; Levenson, J.; Doucette, J.T.; Barbosa, P. Strong Correlations between Empathy, Emotional Intelligence, and Personality Traits among Podiatric Medical Students: A Cross-Sectional Study. Educ. Health Abingdon Engl. 2016, 29, 186-194. [CrossRef]

36. Youssef, F.F.; Nunes, P.; Sa, B.; Williams, S. An Exploration of Changes in Cognitive and Emotional Empathy among Medical Students in the Caribbean. Int. J. Med. Educ. 2014, 5, 185-192. [CrossRef]

37. San-Martín, M.; Roig-Carrera, H.; Villalonga-Vadell, R.M.; Benito-Sevillano, C.; Torres-Salinas, M.; Claret-Teruel, G.; Robles, B.; Sans-Boix, A.; Alcorta-Garza, A.; Vivanco, L. Empathy, inter-professional collaboration, and lifelong medical learning in Spanish and Latin-American physicians-in-training who start their postgraduate training in hospitals in Spain. Preliminary outcomes. Aten. Primaria 2017, 49, 6-12. [CrossRef]

38. Dávila-Pontón, Y.; Reyes-Reyes, A.; Calzadilla-Núñez, A.; Utsman, R.; Torres-Martínez, P.; Díaz-Narváez, V. Empathy and Personality Styles in Medical Students. Rev. Colomb. Psicol. 2020, 29, 73-87. [CrossRef]

39. Ratelle, C.F.; Guay, F.; Vallerand, R.J.; Larose, S.; Senecal, C. Autonomous, Controlled, and Amotivated Types of Academic Motivation: A Person-Oriented Analysis. J. Educ. Psychol. 2007, 99, 734-746. [CrossRef]

40. Doménech-Betoret, F.; Abellán-Roselló, L.; Gómez-Artiga, A. Self-Efficacy, Satisfaction, and Academic Achievement: The Mediator Role of Students' Expectancy-Value Beliefs. Front. Psychol. 2017, 8, 1193. [CrossRef]

41. Webb, A.R.; Young, R.A.; Baumer, J.G. Emotional Intelligence and the ACGME Competencies. J. Grad. Med. Educ. 2010, 2, 508-512. [CrossRef] [PubMed] 
42. Gough, H.G.; Bradley, P.; McDonald, J.S. Performance of Residents in Anesthesiology as Related to Measures of Personality and Interests. Psychol. Rep. 1991, 68, 979-994. [CrossRef]

43. LePine, J.A.; Colquitt, J.A.; Erez, A. Adaptability to Changing Task Contexts: Effects of General Cognitive Ability, Conscientiousness, and Openness to Experience. Pers. Psychol. 2000, 53, 563-593. [CrossRef]

44. Shankar, P.; Dubey, A.; Balasubramanium, R.; Dwivedi, N. Student Attitude towards Communication Skills Learning in a Caribbean Medical School. Australas. Med. J. 2013, 6, 466-475. [CrossRef]

45. Akbarilakeh, M.; Fargah, F.; Razjouyan, K.; Rahimzadeh, M. Predicating Attitude Toward Learning Communication Skills in Medical Students of Shahid Beheshti University. Dis. Diagn. 2020, 9, 21-25. [CrossRef]

46. Gheirati, E.; Shabanifar, A.; Akhlaghi, M.; Peyman, N. Relationship Between Communication Skills and Mental Health Among the Students of Mashhad University of Medical Sciences, Mashhad, Iran. J. Sch. Public Health Inst. Public Health Res. 2016, 14, 61-72.

47. Nami, K.; Ghalavandi, H.; Hosseinpoor, A.R. The Role of Students Personality Traits on Students Learning Style in University of Medical Sciences. Dev. Strateg. Med. Educ. 2014, 1, 59-68.

48. Molinuevo, B.; Torrubia, R. Does Personality Predict Medical Students' Attitudes to Learning Communication Skills? Int. J. Med. Educ. 2013, 4, 155-161. [CrossRef]

49. Zare-Alamshiri, M.; Saber, S. Prediction of Communication Skills Based on Psycho-Social Class Atmosphere and Social Anxiety of High School Students. Community Health 2017, 4, 127-136.

50. O'Tuathaigh, C.M.P.; Nadhirah Idris, A.; Duggan, E.; Costa, P.; Costa, M.J. Medical Students' Empathy and Attitudes towards Professionalism: Relationship with Personality, Specialty Preference and Medical Programme. PLoS ONE 2019, 14, e0215675. [CrossRef]

51. Sims, C.M. Do the Big-Five Personality Traits Predict Empathic Listening and Assertive Communication? Int. J. Listening 2017, 31, 163-188. [CrossRef]

52. Kuntze, J.; van der Molen, H.T.; Born, M.P. Big Five Personality Traits and Assertiveness Do Not Affect Mastery of Communication Skills. Health Prof. Educ. 2016, 2, 33-43. [CrossRef]

53. Franco, C.A.G.d.S.; Franco, R.S.; Cecilio-Fernandes, D.; Severo, M.; Ferreira, M.A. The Assessment of Personality Traits and Its Association with Learning Communication Skills. Sci. Med. Porto Alegre Online 2020, 30, 37326.

54. Vermetten, Y.J.; Lodewijks, H.G.; Vermunt, J.D. The Role of Personality Traits and Goal Orientations in Strategy Use. Contemp. Educ. Psychol. 2001, 26, 149-170. [CrossRef] [PubMed]

55. Holen, A.; Manandhar, K.; Pant, D.S.; Karmacharya, B.M.; Olson, L.M.; Koju, R.; Mansur, D.I. Medical Students' Preferences for Problem-Based Learning in Relation to Culture and Personality: A Multicultural Study. Int. J. Med. Educ. 2015, 6, 84-92. [CrossRef]

56. Tsou, K.-I.; Lin, C.-S.; Cho, S.-L.; Powis, D.; Bore, M.; Munro, D.; Sze, D.M.-Y.; Wu, H.-C.; Hsieh, M.-S.; Lin, C.-H. Using Personal Qualities Assessment to Measure the Moral Orientation and Personal Qualities of Medical Students in a Non-Western Culture. Eval. Health Prof. 2013, 36, 174-190. [CrossRef]

57. Costa, P.T.; McCrae, R.R. Revised NEO Personality Inventory (NEO PI-R) and NEO Five-Factor Inventory (NEO-FFI); Psychological Assessment Resources: Odessa, FL, USA, 1992.

58. Saucier, G.; Hampson, S.; Goldberg, L. Cross-language Studies of Lexical Personality Factors. In Advances in Personality Psychology; Hampson, S.E., Ed.; Psychology Press: Philadelphia, PA, USA, 2000; Volume 1, pp. 1-36.

59. John, O.; Pervin, R.; Pervin, L. The Five-Factor Theory of Personality. In Handbook of Personality: Theory and Research; McCrae, R., Costa, P.J., Eds.; Guilford Press: New York, NY, USA, 2008; p. 171.

60. Sava, F. Inventarul de Personalitate DECAS; ArtPress: Timişoara, Romania, 2008.

61. Extraversion and Happiness: The Mediating Role of Social Support and Hope-PubMed. Available online: https://pubmed.ncbi. nlm.nih.gov/30015375/ (accessed on 29 June 2021).

62. Bennett, K.L.; Phillips, J.P. Finding, Recruiting, and Sustaining the Future Primary Care Physician Workforce: A New Theoretical Model of Specialty Choice Process. Acad. Med. J. Assoc. Am. Med. Coll. 2010, 85, S81-S88. [CrossRef]

63. Thomas, J.H. The Surgical Personality: Fact or Fiction. Am. J. Surg. 1997, 174, 573-577. [CrossRef]

64. Zwarenstein, M.; Goldman, J.; Reeves, S. Interprofessional Collaboration: Effects of Practice-Based Interventions on Professional Practice and Healthcare Outcomes. Cochrane Database Syst. Rev. 2009, CD000072. [CrossRef]

65. Stoller, J.K.; Taylor, C.A.; Farver, C.F. Emotional Intelligence Competencies Provide a Developmental Curriculum for Medical Training. Med. Teach. 2013, 35, 243-247. [CrossRef]

66. Petrides, K. Technical Manual for the Trait Emotional Intelligence Questionnaires (TEIQue), 1st ed.; 2nd Printing; London Psychometric Laboratory: London, UK, 2009.

67. Cherry, M.G.; Fletcher, I.; O'Sullivan, H.; Shaw, N. What Impact Do Structured Educational Sessions to Increase Emotional Intelligence Have on Medical Students? BEME Guide No. 17. Med. Teach. 2012, 34, 11-19. [CrossRef]

68. Johnson, D.R. Emotional Intelligence as a Crucial Component to Medical Education. Int. J. Med. Educ. 2015, 6, 179-183. [CrossRef] [PubMed]

69. Zaki, J. Empathy: A Motivated Account. Psychol. Bull. 2014, 140, 1608-1647. [CrossRef]

70. Goleman, D. Emotional Intelligence: Why It Can Matter More than IQ; Bantam Books: New York, NY, USA, 1995. 
71. ECFMG-ACGME Webinar: ACGME Core Competencies and Milestones. Available online: https://www.ecfmg.org/echo/ webinars-acgme-core-competencies-2018.html (accessed on 22 September 2021).

72. Arora, S.; Ashrafian, H.; Davis, R.; Athanasiou, T.; Darzi, A.; Sevdalis, N. Emotional Intelligence in Medicine: A Systematic Review through the Context of the ACGME Competencies. Med. Educ. 2010, 44, 749-764. [CrossRef] [PubMed]

73. Barrick, M.R.; Mount, M.K. The Big Five Personality Dimensions and Job Performance: A Meta-Analysis. Pers. Psychol. 1991, 44, 1-26. [CrossRef]

74. Burch, G.; Anderson, N. Personality as a Predictor of Working-Related Behavior and Performance: Recent Advances and Directions for Future Research. In International Review of Industrial and Organizational Psychology; Hodgkinson, G., Ford, J., Eds.; Wiley-Blackwell: Chichester, UK, 2008; pp. 261-305.

75. The AAMC Project on the Clinical Education of Medical Students-MOAM.INFO. Available online: https://moam.info/theaamc-project-on-the-clinical-education-of-medical-students_59fa642f1723dd0b1e9cf133.html (accessed on 25 September 2021).

76. Palsson, R.; Kellett, J.; Lindgren, S.; Merino, J.; Semple, C.; Sereni, D.; EFIM/UEMS Working Group on Competencies in Internal Medicine in Europe. Core Competencies of the European Internist: A Discussion Paper. Eur. J. Intern. Med. 2007, 18, 104-108. [CrossRef] [PubMed] 